\title{
Strong SUSY at ATLAS and CMS
}

Nadja Strobbe (Fermilab)

On behalf on the ATLAS and CMS Collaborations Moriond QCD 2019
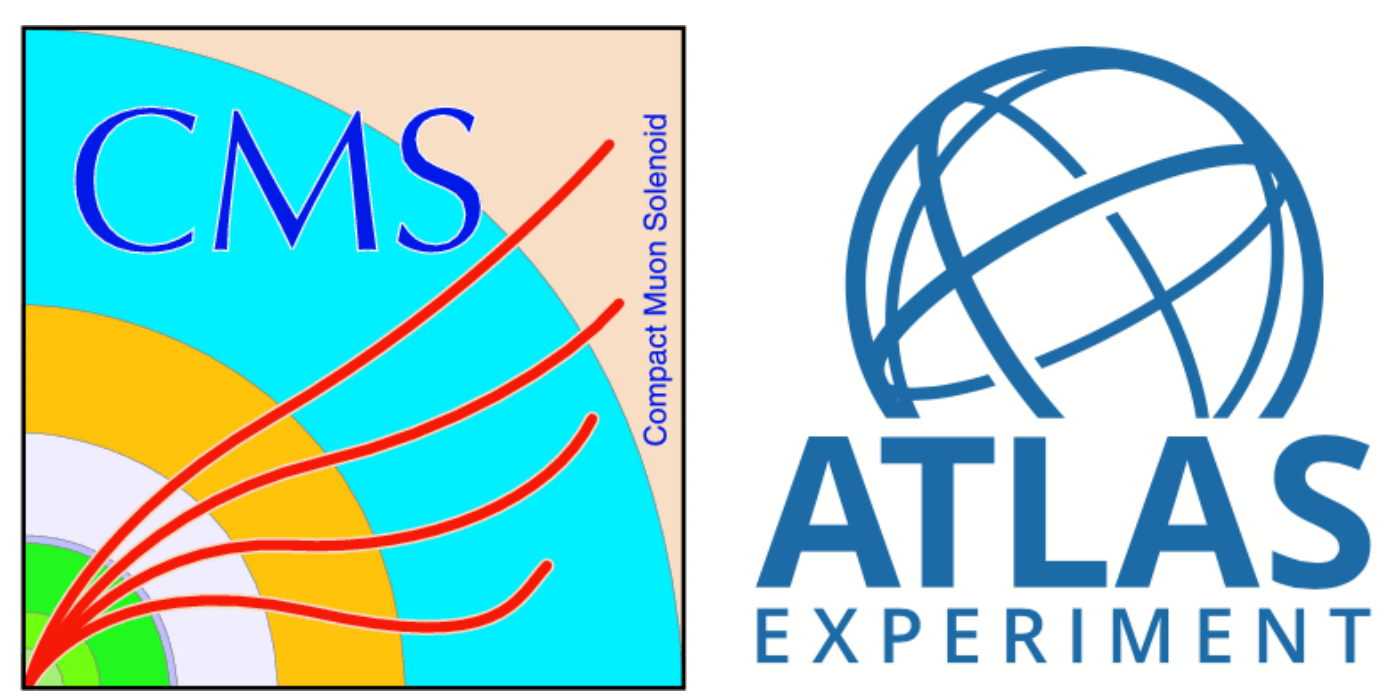

This document was prepared by [ATLAS AND CMS Collaborations] using the resources of the Fermi National Accelerator Laboratory (Fermilab), a U.S.

Department of Energy, Office of Science, HEP User Facility. Fermilab is managed by Fermi Research Alliance, LLC (FRA), acting under Contract No. DEAC02-07CH11359.
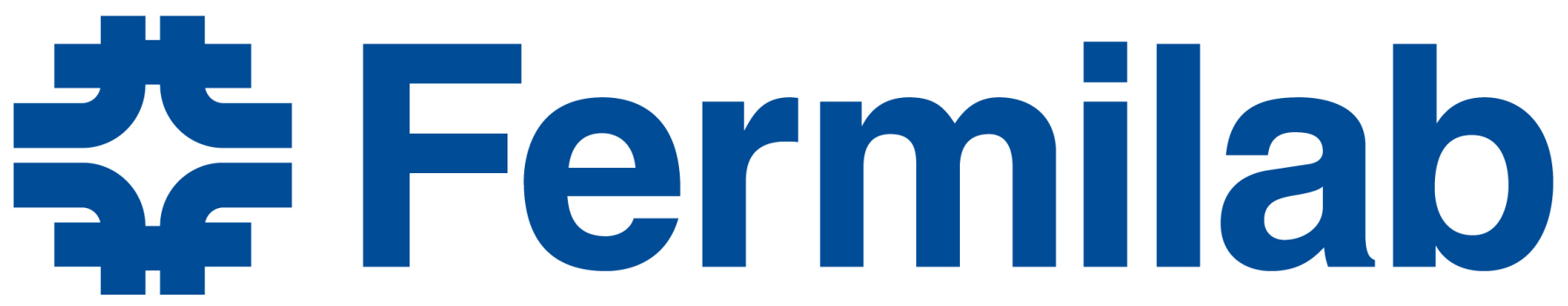


\section{Introduction}

- LHC performed very well, delivering $>150 \mathrm{fb}^{-1}$

- Huge challenge to quickly process this amount of data

- Understand detector \& object performance

- $\mathrm{pT}^{\text {miss }}$ tails very important for SUSY searches

- Most results on full Run 2 data set will come in Summer

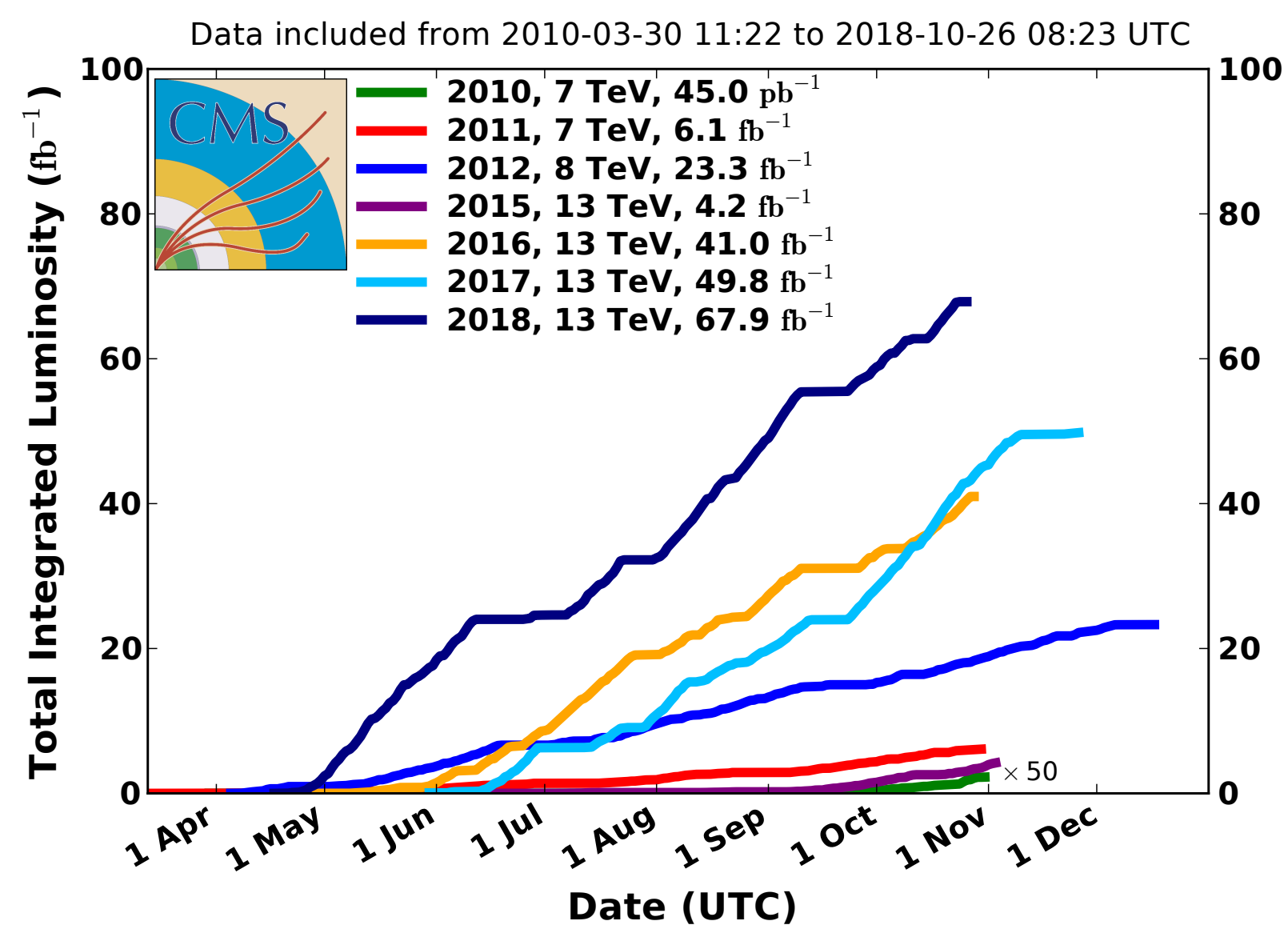

- SUSY searches are expanding

- Big inclusive searches complemented by dedicated searches to close gaps in coverage

- Machine learning for $\mathrm{H}, \mathrm{b}, \mathrm{c}$, top,... tagging

- Improved analysis techniques 


\section{Overview of recent Strong SUSY results}

- ATLAS

- bjets+H+p $T^{m i s s}\left(139 \mathrm{fb}^{-1}\right)$

- Displaced vertex/muon (136 fb-1)

- Stop via té spin correlations $\left(36 \mathrm{fb}^{-1}\right)$

ATLAS-CONF-2019-011

- $\geq 3 b+p_{T^{m i s s}}\left(80 \mathrm{fb}^{-1}\right)$

- Stop with c tagging $\left(36 \mathrm{fb}^{-1}\right)$

- $T_{h}+$ jets $+\mathrm{p}_{T^{m i s s}}\left(36 \mathrm{fb}^{-1}\right)$

ATLAS-CONF-2019-006

arxiv.org: 1903.07570

ATLAS-CONF-2018-041

arxiv:1805.01649

arxiv: 1808.06358

- CMS

- Jets + MT2 $\left(137 \mathrm{fb}^{-1}\right)$

- Same-sign $2 \mathrm{l}+\geq 3 \mathrm{l}\left(137 \mathrm{fb}^{-1}\right)$

- GGM combination (36 fb-1)

- 2 photons $+\mathrm{pT}^{\text {miss }}\left(36 \mathrm{fb}^{-1}\right)$

- (b)jets + photon $\left(36 \mathrm{fb}^{-1}\right)$

- Stop in top corridor (36 fb-1)

CMS-PAS-SUS-19-005

CMS-PAS-SUS-19-008

CMS-PAS-SUS-18-005

arxiv:1903.07070

arxiv: 1901.06726

arxiv:1901.01288

- ATLAS public results page: https://twiki.cern.ch/twiki/bin/view/AtlasPublic/ SupersymmetryPublicResults

- CMS public results page: http://cms-results.web.cern.ch/cms-results/public-results/ publications/SUS/index.html 
- Inclusive search targeting wide range of strong SUSY models

- 282 signal regions defined using $\mathrm{H}_{\mathrm{T}}, \mathrm{N}_{\mathrm{j}}, \mathrm{N}_{\mathrm{b}}, \mathrm{M}_{\mathrm{T} 2}$

NEW disappearing track selection covered by K. Pedro

- Backgrounds estimated using data control regions

- Lost lepton: $\mathrm{PT}^{\text {miss }}$ from $\mathrm{W} \rightarrow \mathrm{IV}$

- Irreducible, $\mathrm{p}_{T}{ }^{\text {miss }}$ from $\mathrm{Z} \rightarrow \mathrm{vV}$

- $\mathrm{p}_{\mathrm{T}}^{\text {miss }}$ from jet mismeasurements

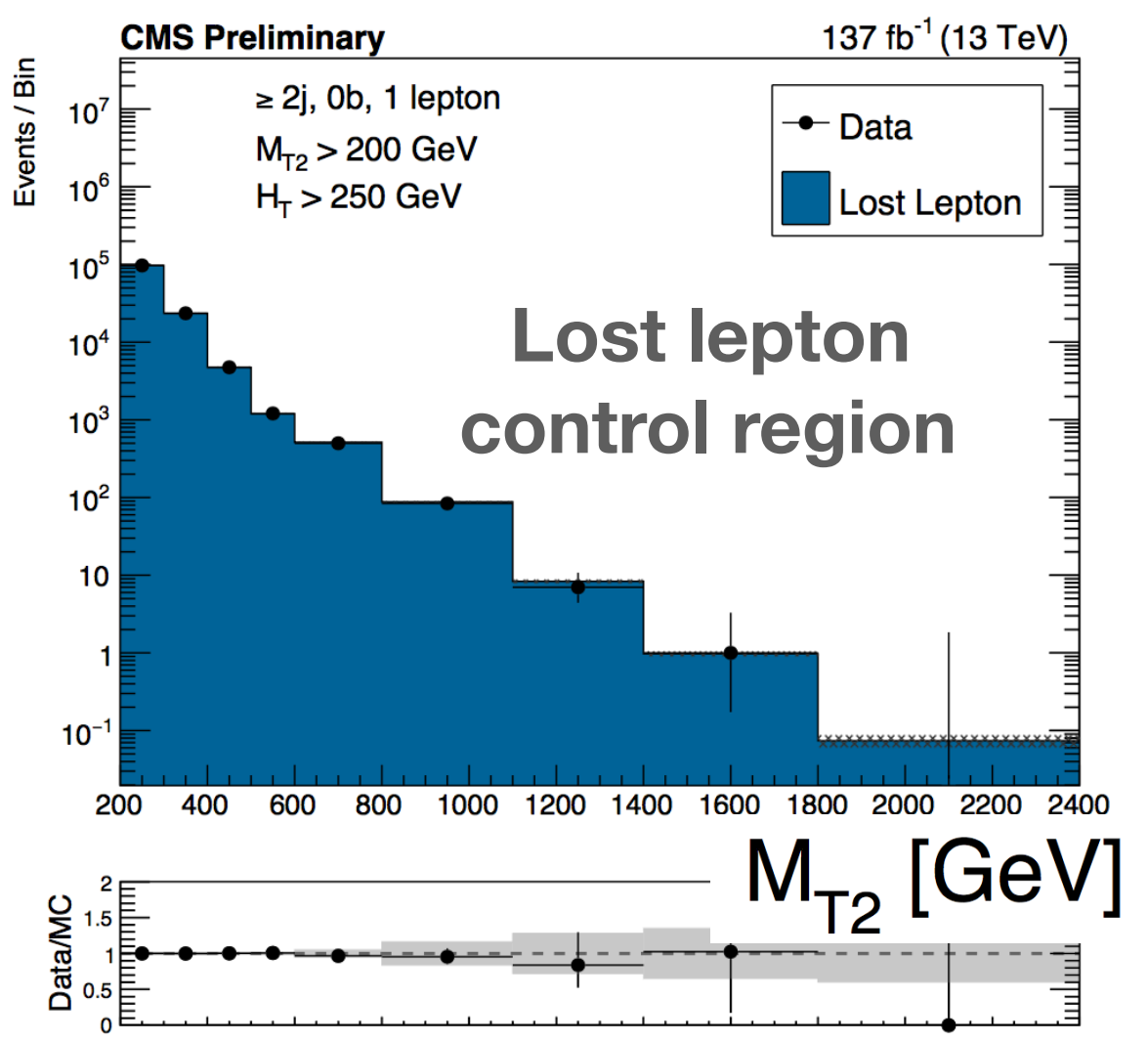

We observe no evidence for an excess above the expected background event yield 


\section{Jets $+\mathrm{M}_{\mathrm{T} 2}$}

- Interpret using simplified models of squark and gluino production

- Extend reach by $150-350 \mathrm{GeV}$ compared to $36 \mathrm{fb}^{-1}$ results

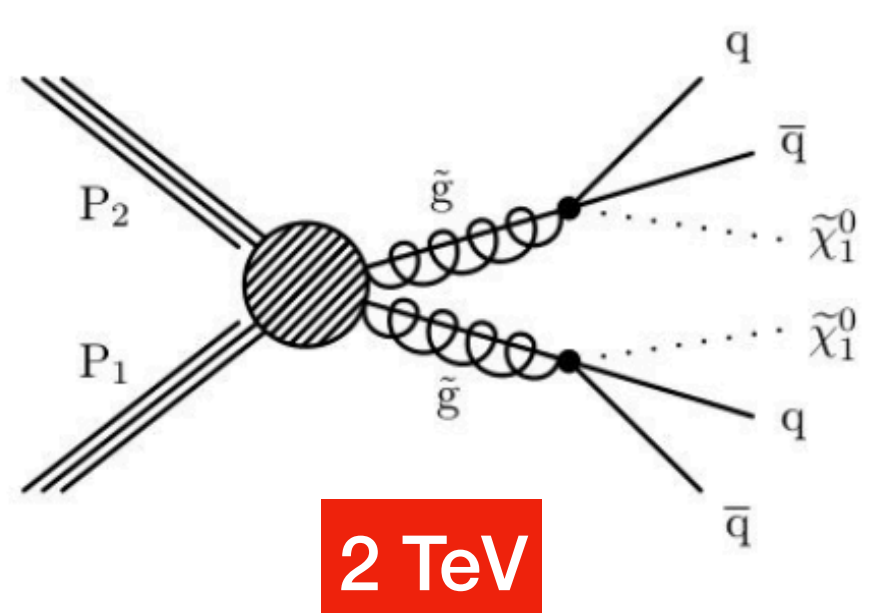

CMS Preliminary $137 \mathrm{fb}^{-1}(13 \mathrm{TeV})$

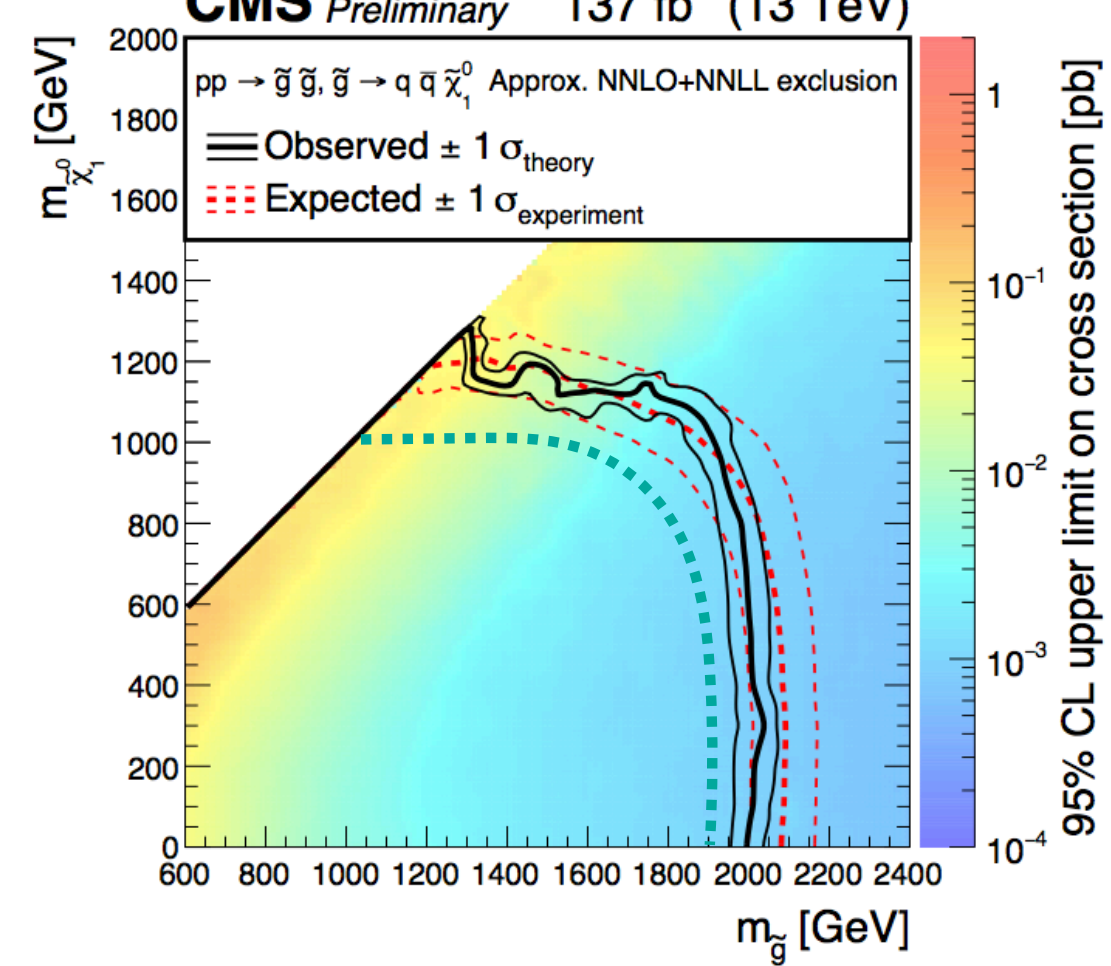

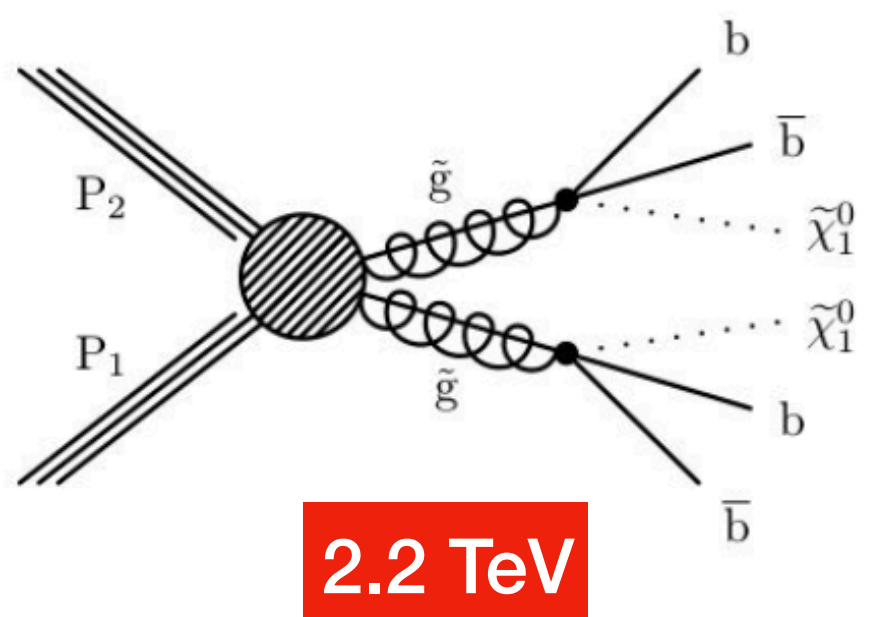

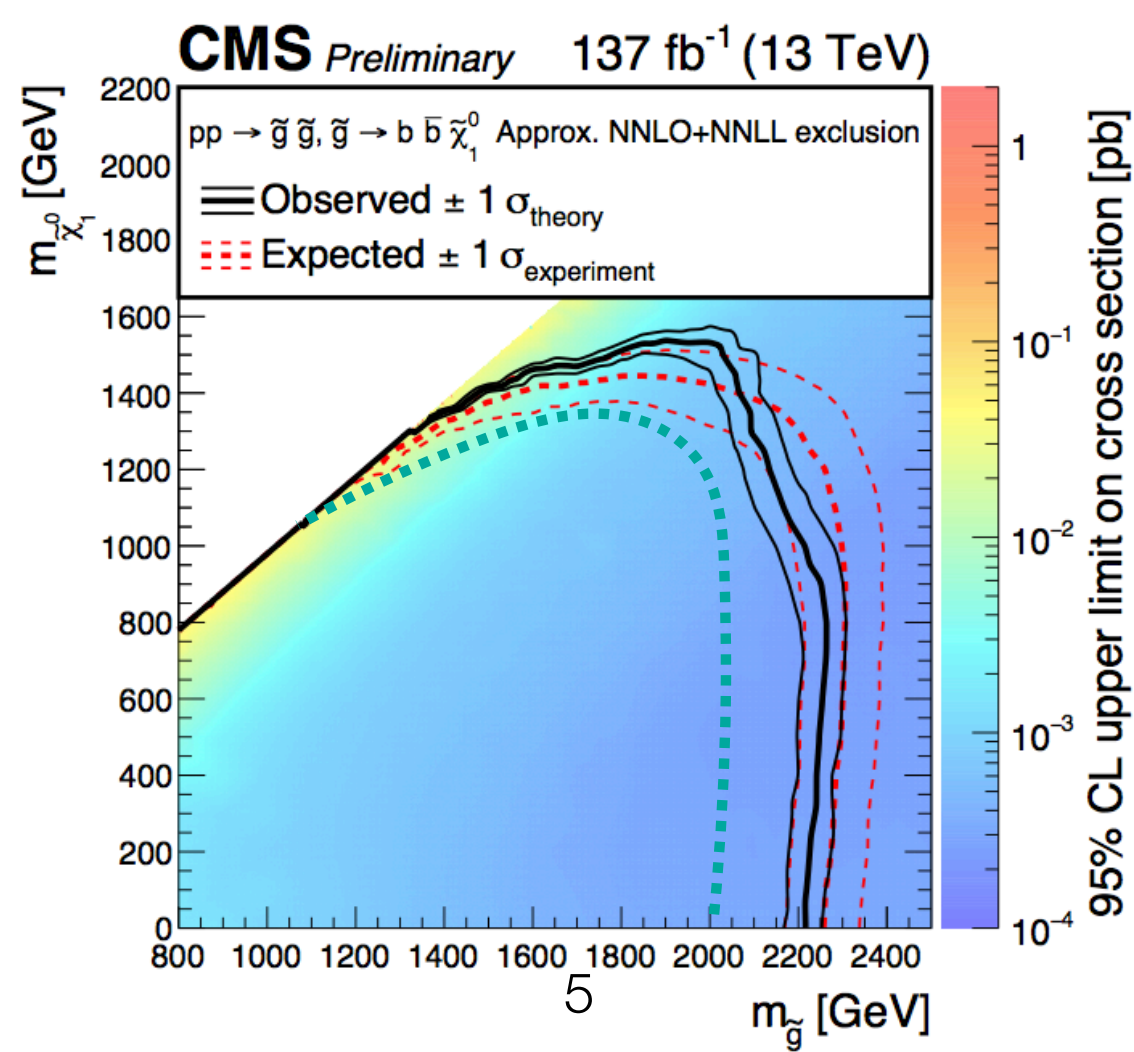

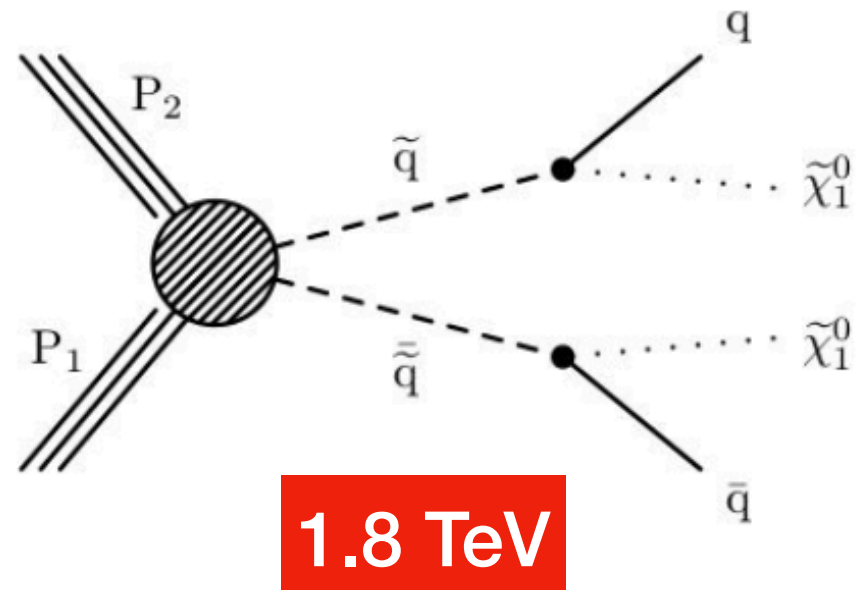

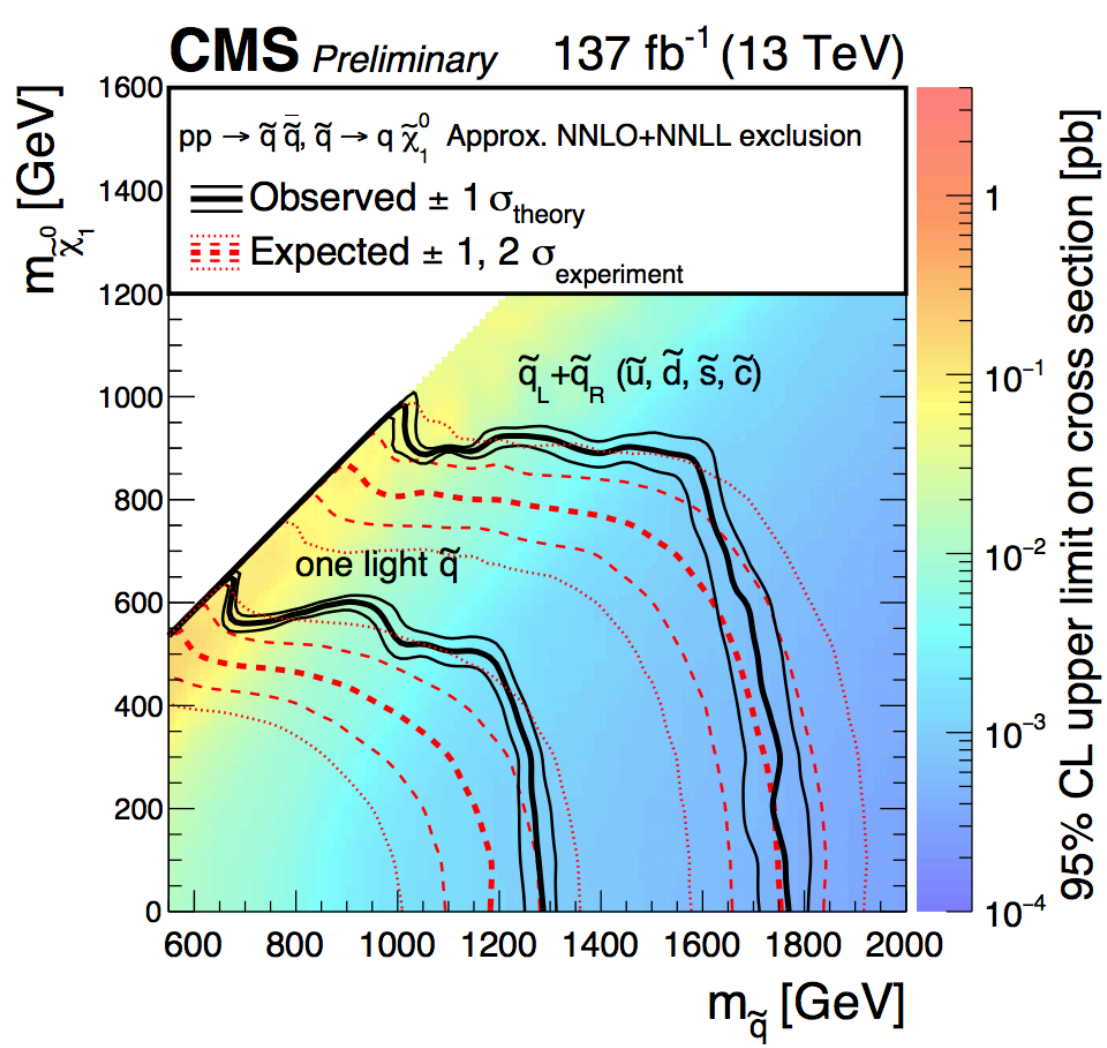


- Use Higgs boson as probe for new physics

- Exploit large branching fraction of $\mathrm{H} \rightarrow b \bar{b}$

- Use $\Delta R$ and invariant mass of Higgs candidates

- Search regions with many b-jets and p $^{\text {miss }}$

- Optimized to target different regions of phase space, e.g. bulk vs compressed

- Main background is $t \bar{t}$ or $Z+j e t s$, estimated using 1- and 2-lepton data control regions
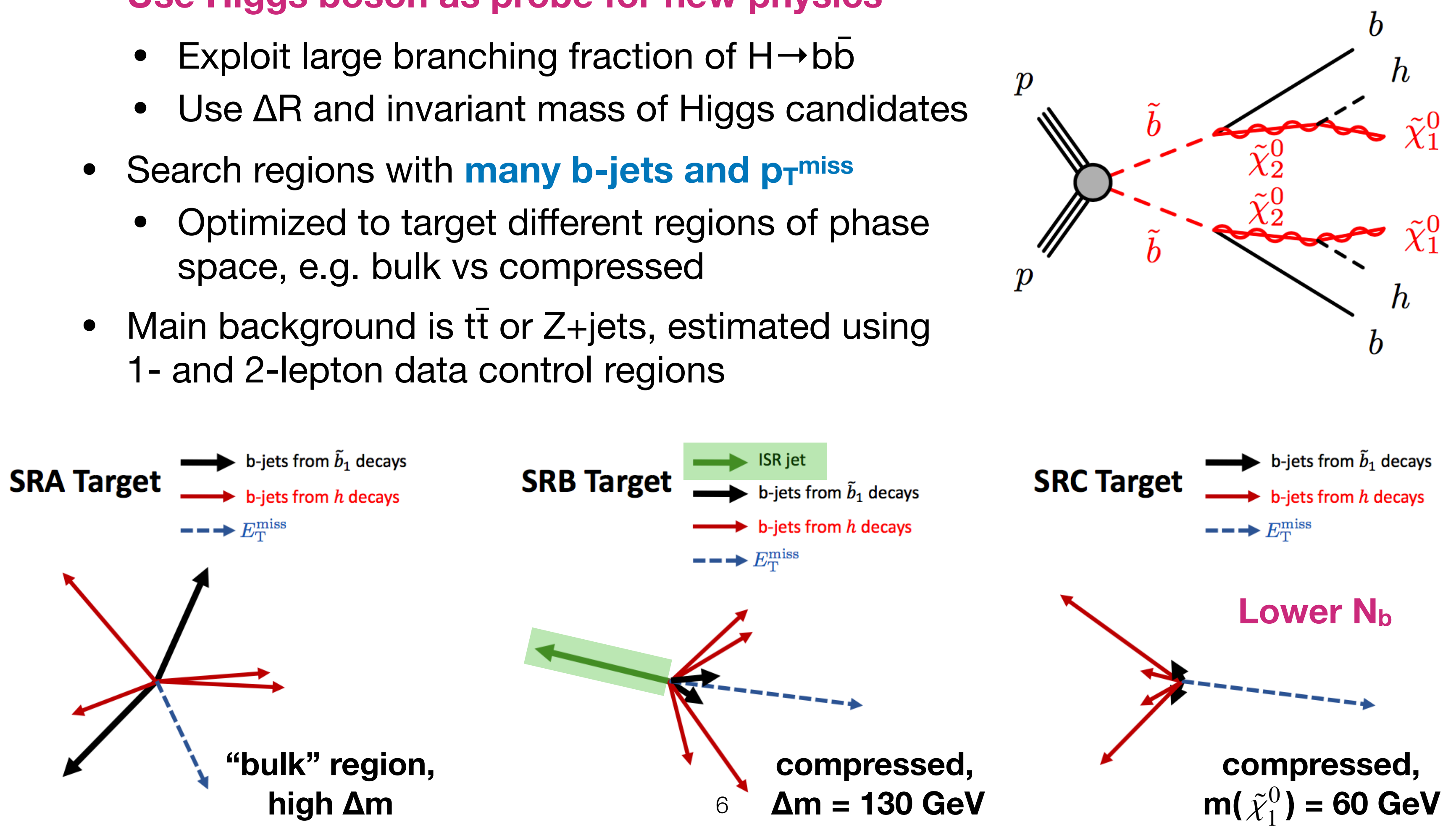


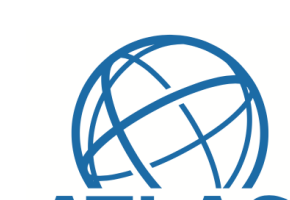
ATLAS

\section{bjets $+\mathrm{H}$}
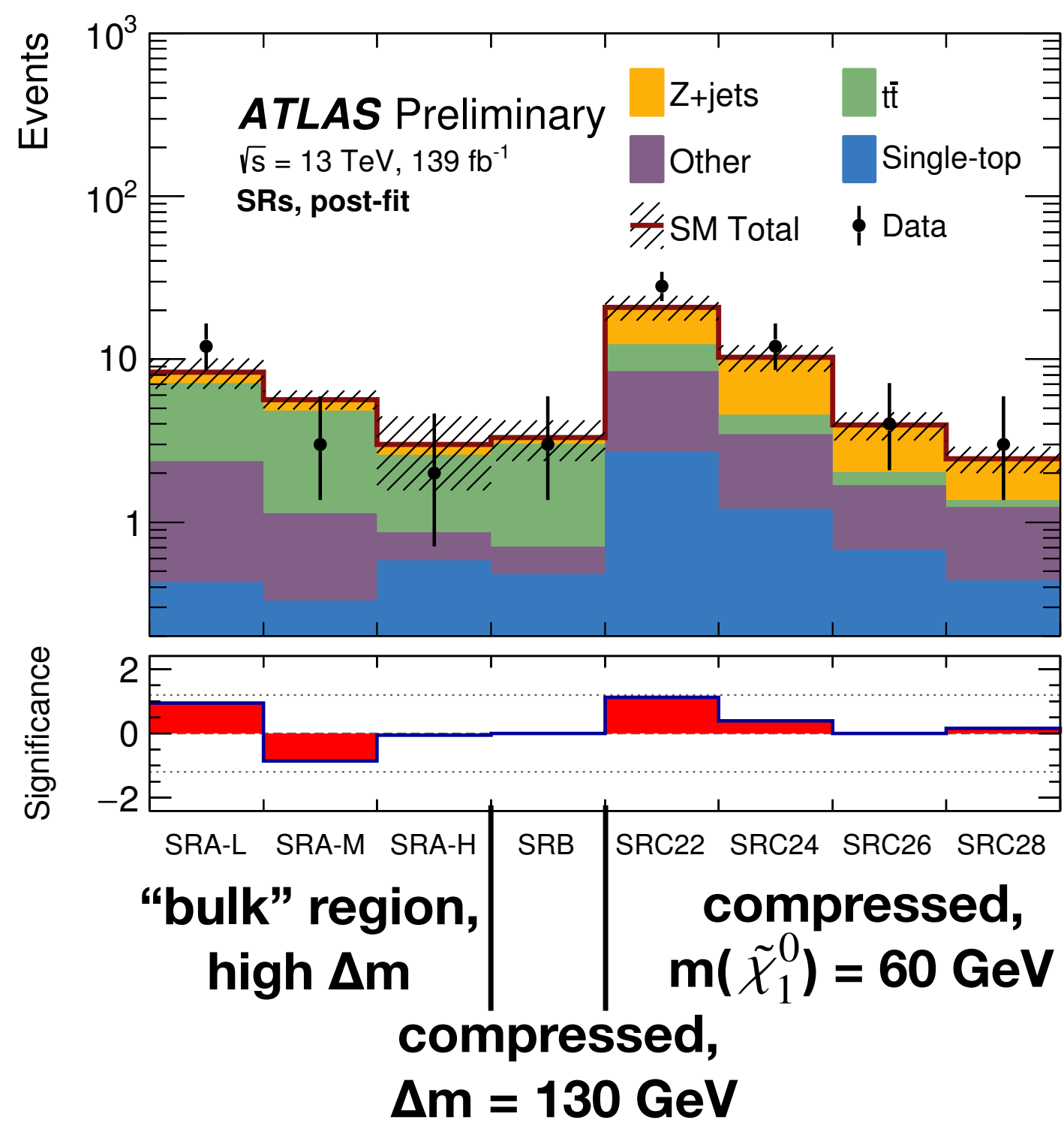

- Sbottom quarks excluded up to $1400 \mathrm{GeV}$ for $m\left(\tilde{\chi}_{1}^{0}\right)=60 \mathrm{GeV}$

- Sensitivity reduced when Higgs boson is just barely on shell
$+\mathrm{p}_{T^{\text {miss }}}$

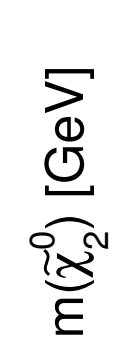

$\tilde{b}_{1} \tilde{b}_{1}$ production $; \tilde{b}_{1} \rightarrow b \tilde{\chi}_{2}^{0} \rightarrow b h \tilde{x}_{1}^{0} ; \Delta \mathrm{m}\left(\tilde{x}_{2}^{0}, \tilde{x}_{1}^{0}\right)=130 \mathrm{GeV}$
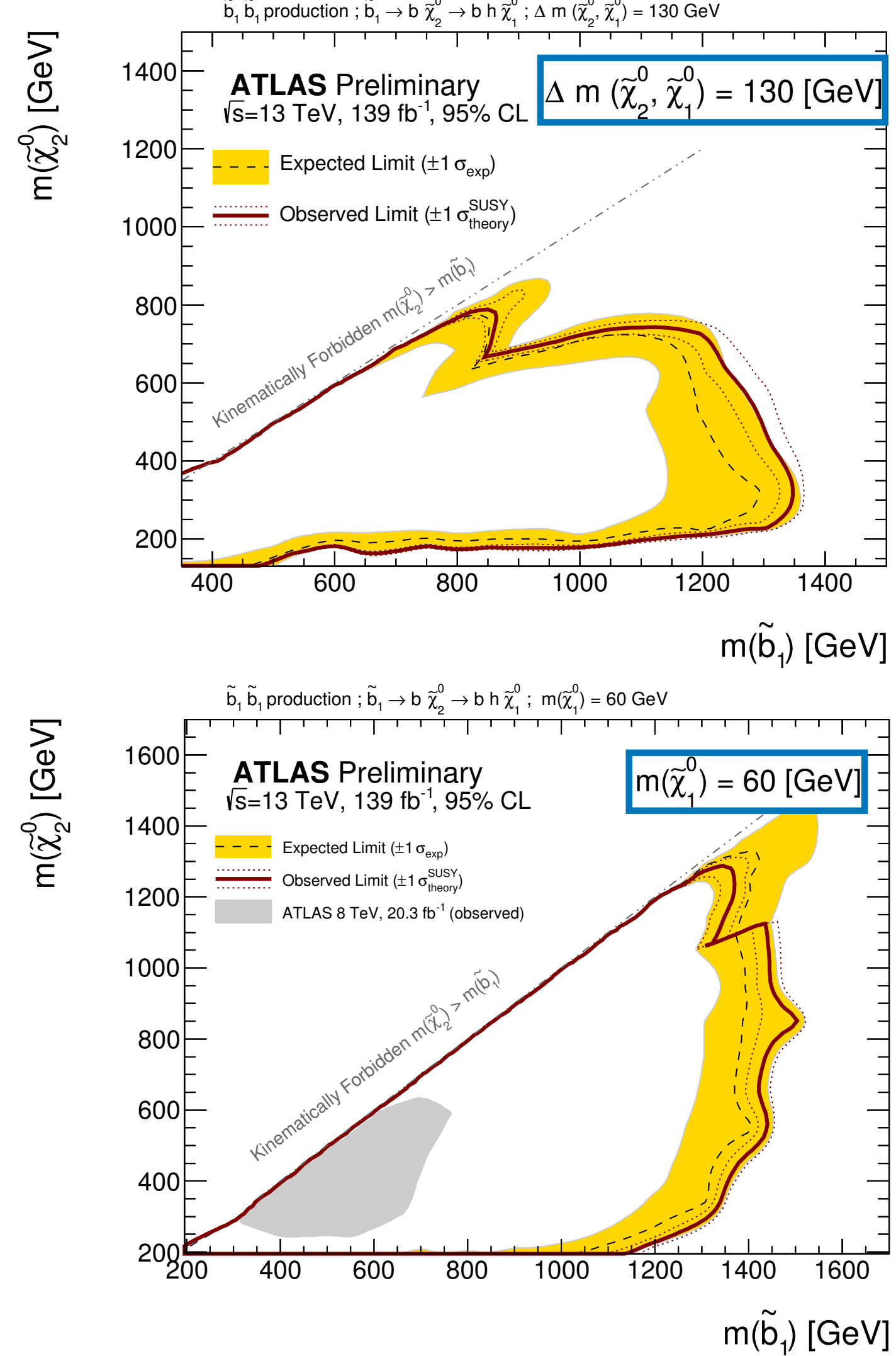
- Processes producing 2 same-sign or $\geq 3$ leptons very rare in SM $\rightarrow$ Low-background environment for searches

- Broad search targeting gluinos, top squarks, bottom squarks

- 168 search regions defined using kinematic variables: lepton PT, $\mathrm{N}_{\mathrm{j}}, \mathrm{N}_{\mathrm{b}}, \mathrm{m}_{\mathrm{T}}^{\mathrm{min}}, \mathrm{H}_{\mathrm{T}}, \mathrm{p}_{\mathrm{T}^{\mathrm{miss}}}$, lepton charge

- Includes regions at low $\mathrm{p}^{\mathrm{m}}$ iss $\rightarrow$ sensitivity to RPV models

- Main backgrounds:

- Rare SM, e.g. WZ, ttW, ...

- Nonprompt leptons

- Electron charge flip

- Good agreement between data and prediction is observed

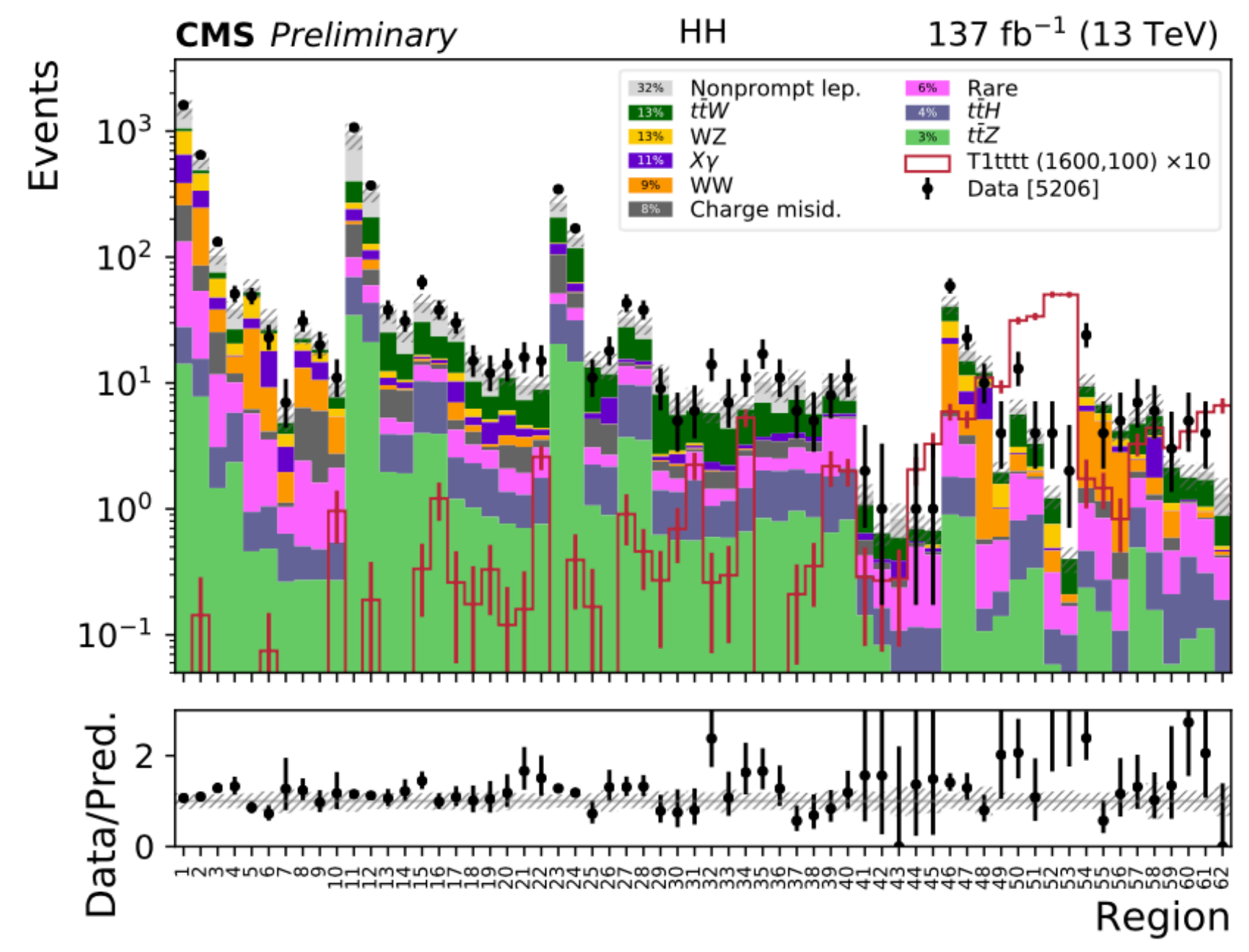




\section{SS + multilepton}

- Reach extended by $150-200 \mathrm{GeV}$ compared to $36 \mathrm{fb}^{-1}$
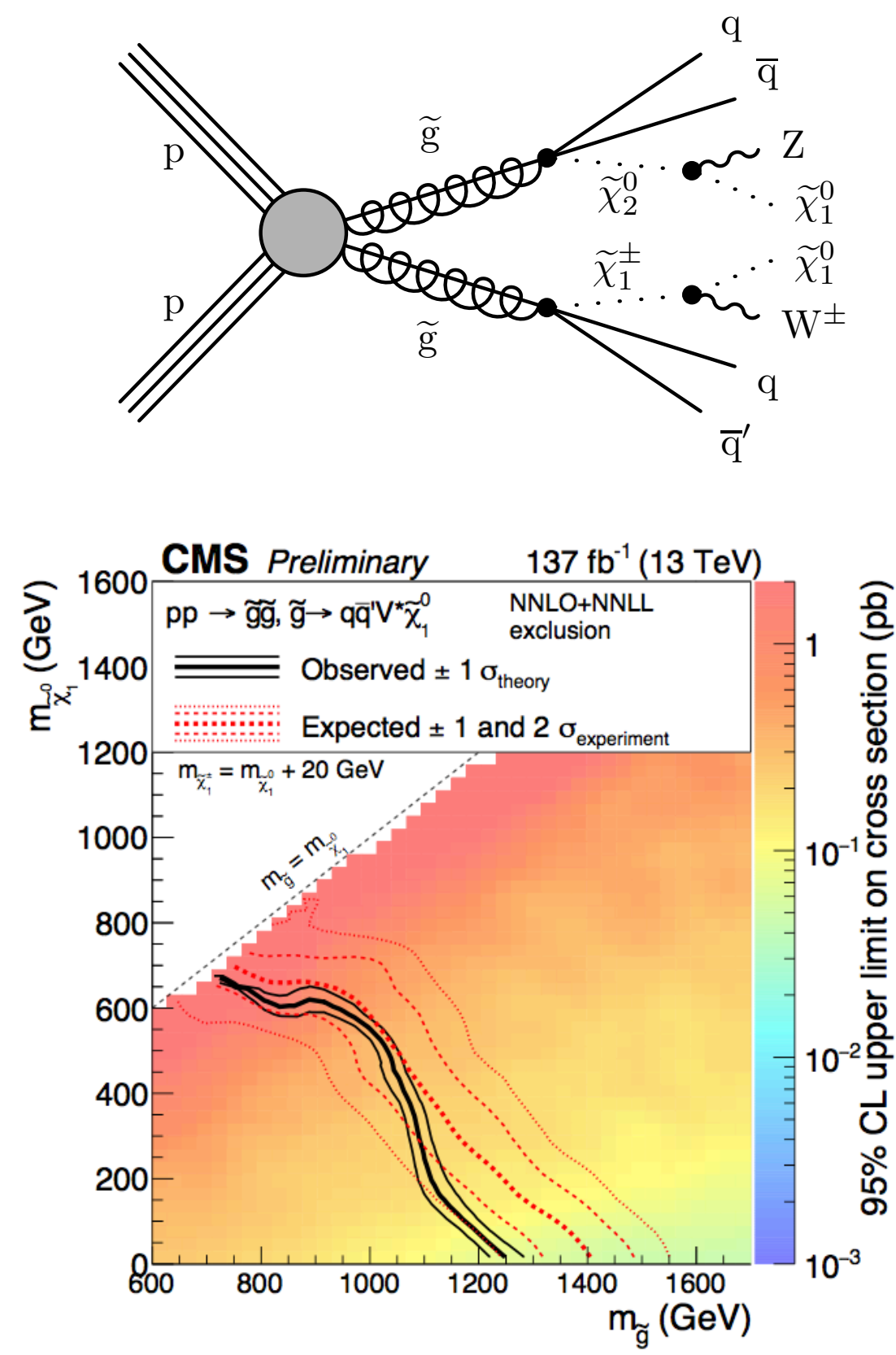
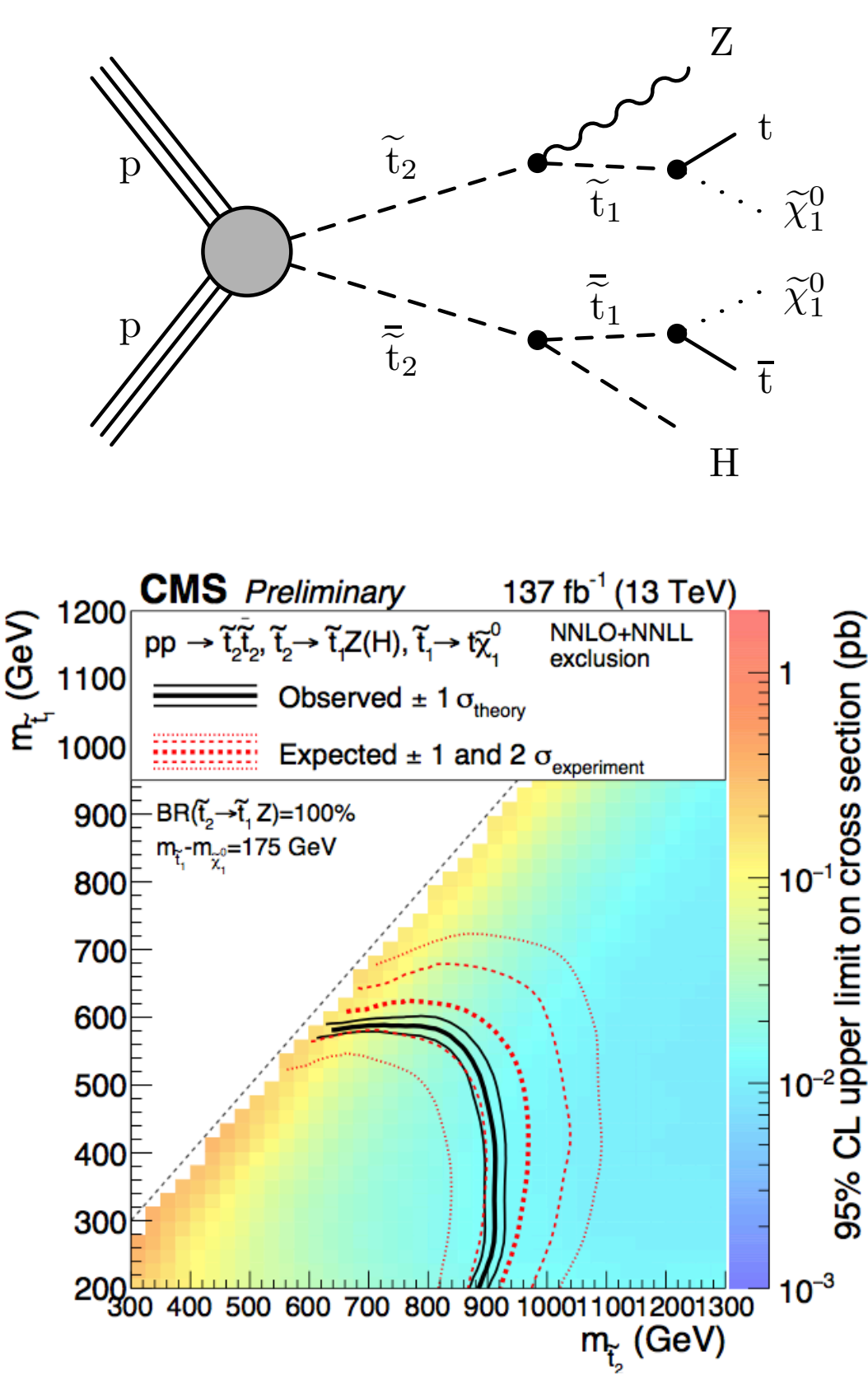

New interpretation!

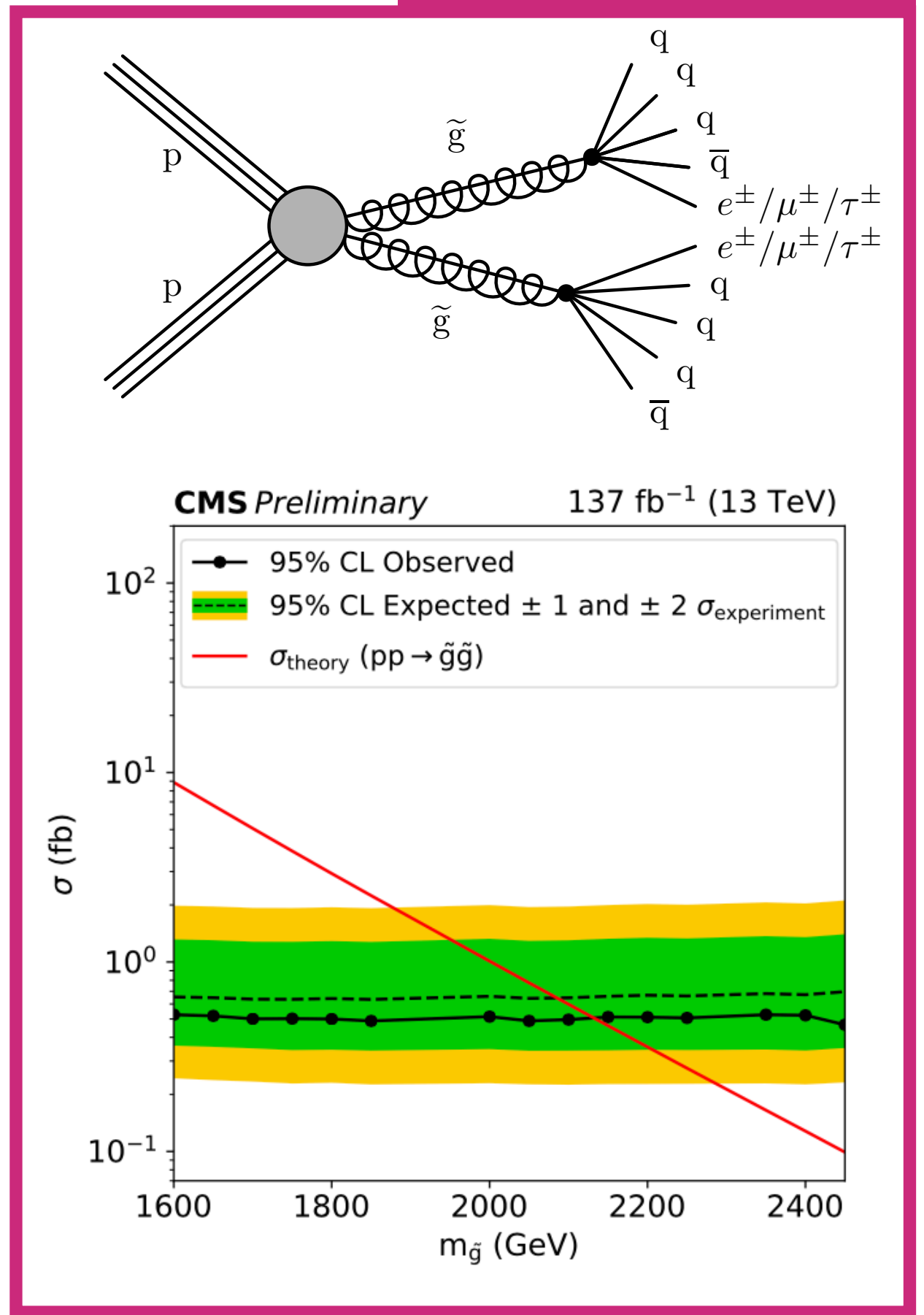




\section{Top squarks}

- Recent results with partial data set focusing on tricky corners of phase space

- 'Compressed spectra' with charm tagging and high $\mathrm{p}^{\mathrm{miss}}$ (from ISR boost)

- Also sensitive to charm squark production (up to $800 \mathrm{GeV}$ )
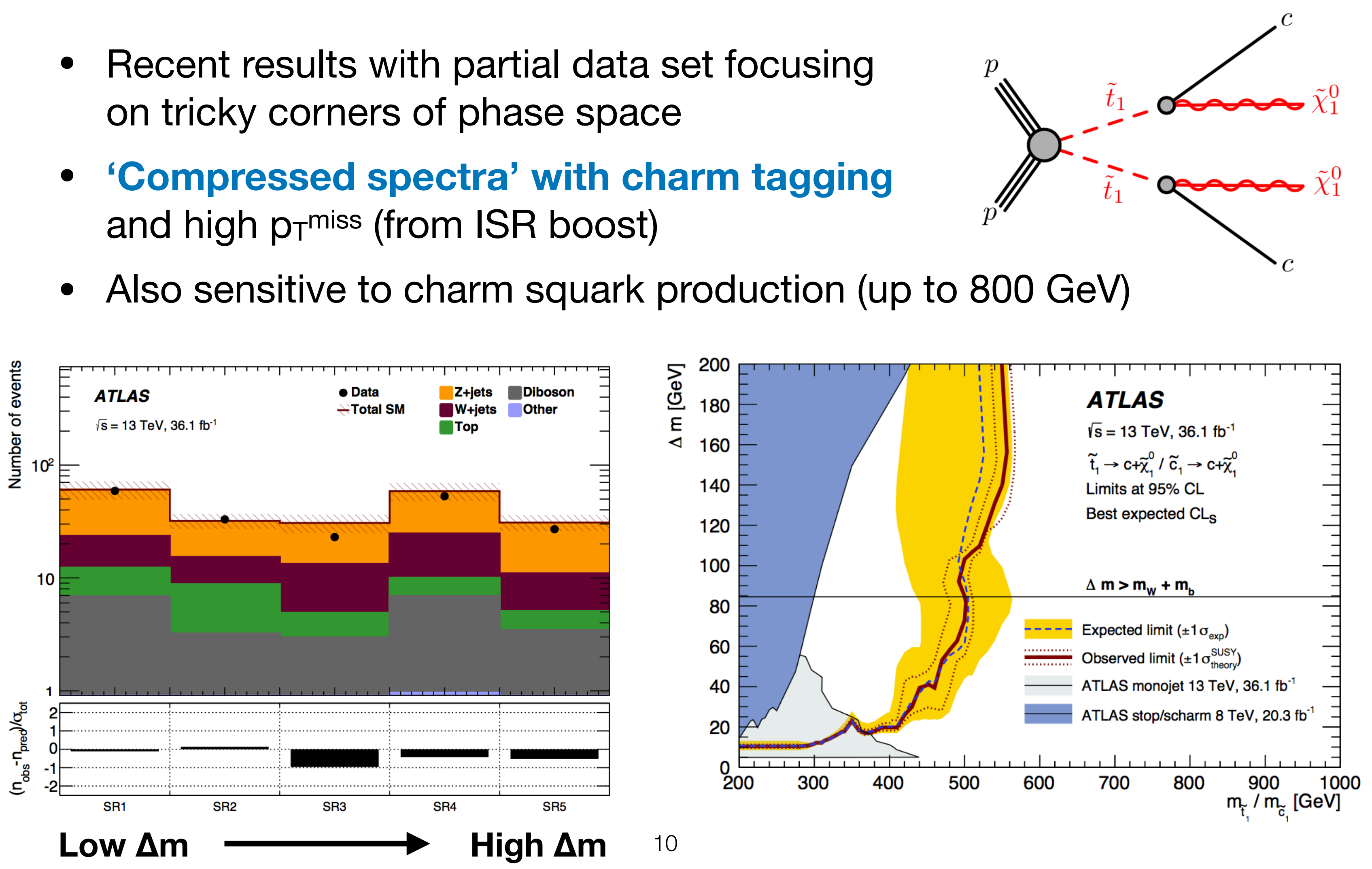
- Motivated by gauge-mediated SUSY breaking

- Combination of 4 analyses, overlaps removed:

- Photon + lepton + $\mathrm{pT}^{\text {miss }}$

- Photon $+\mathrm{ST}_{\mathrm{T}}+\mathrm{p}_{\mathrm{T}^{\mathrm{miss}}}$

- Photon $+\mathrm{H}_{\mathrm{T}}+\mathrm{p}_{\mathrm{T}^{\text {miss }}}$

- Diphoton $+\mathrm{p}^{\mathrm{miss}}$ (not included for $\tilde{g} \tilde{g}$ interpretation)

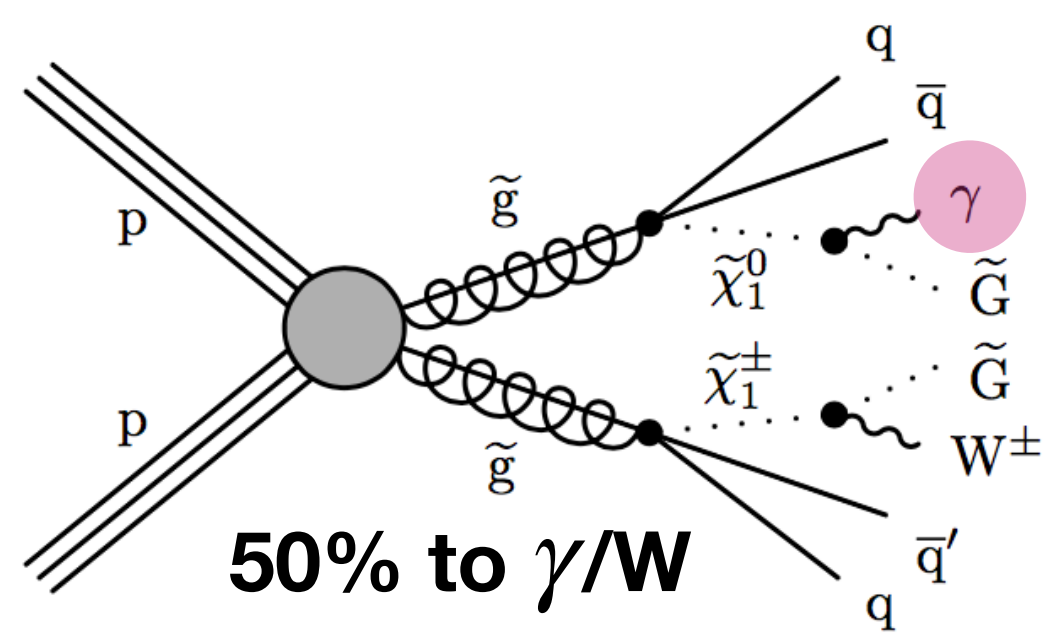

Sensitivity extended in compressed region
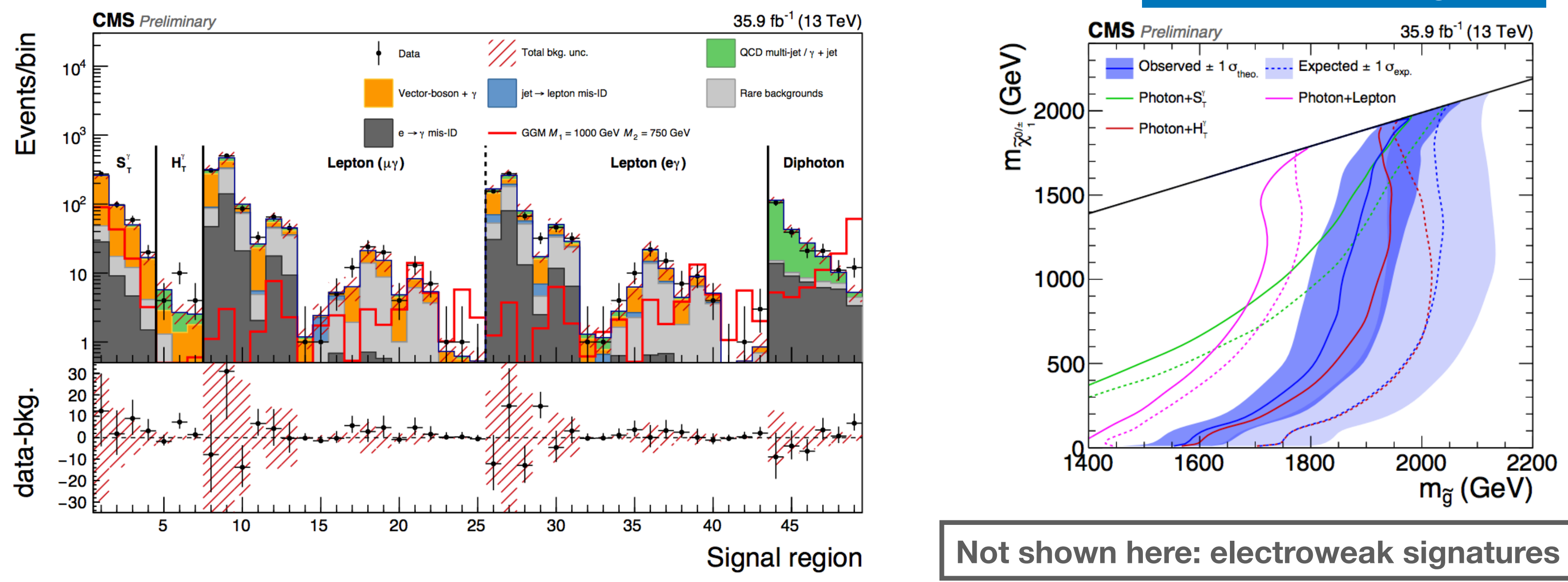

Not shown here: electroweak signatures 


\section{Summary \& outlook}

- Successful Run 2 of the LHC finished at the end of 2018

- Now in LS2, preparing for Run 3

- First full Run 2 results on strong SUSY presented

- Exclude gluinos up to $2.2 \mathrm{TeV}$ and sbottoms up to $1.4 \mathrm{TeV}$ in simplified models

- Many more results to come in next months

- Dedicated analyses with slightly longer time scales

- Results with 2016+2017 data still appearing

- Going after difficult corners of phase space and closing gaps

- Not done yet! HL-LHC data set allows for increase in sensitivity of $0.5-1 \mathrm{TeV}$ 


\section{Backup}

To be filled out 


\section{Jets $+\mathrm{M}_{\mathrm{T} 2}$}

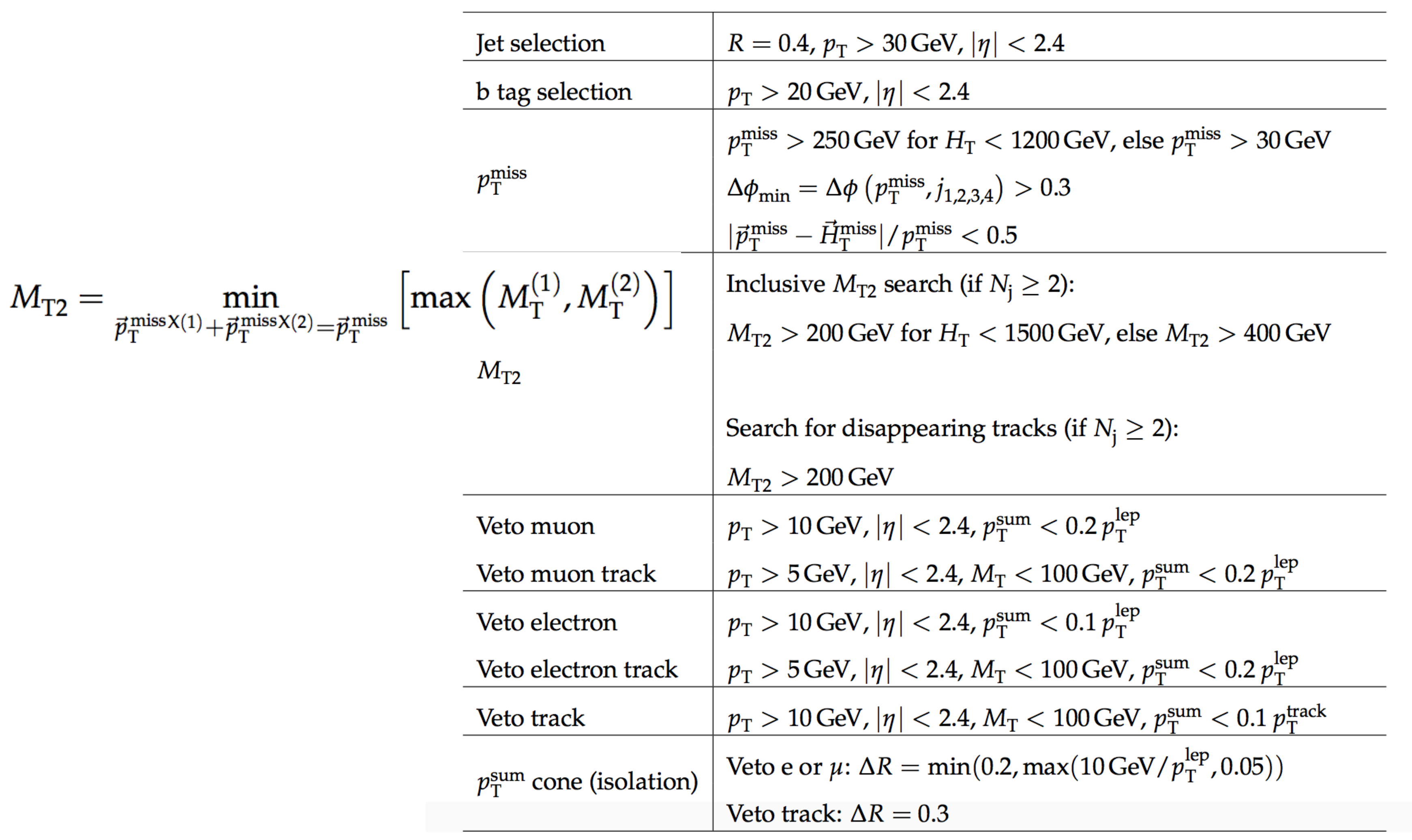




\section{Jets $+\mathrm{M}_{\mathrm{T} 2}$}
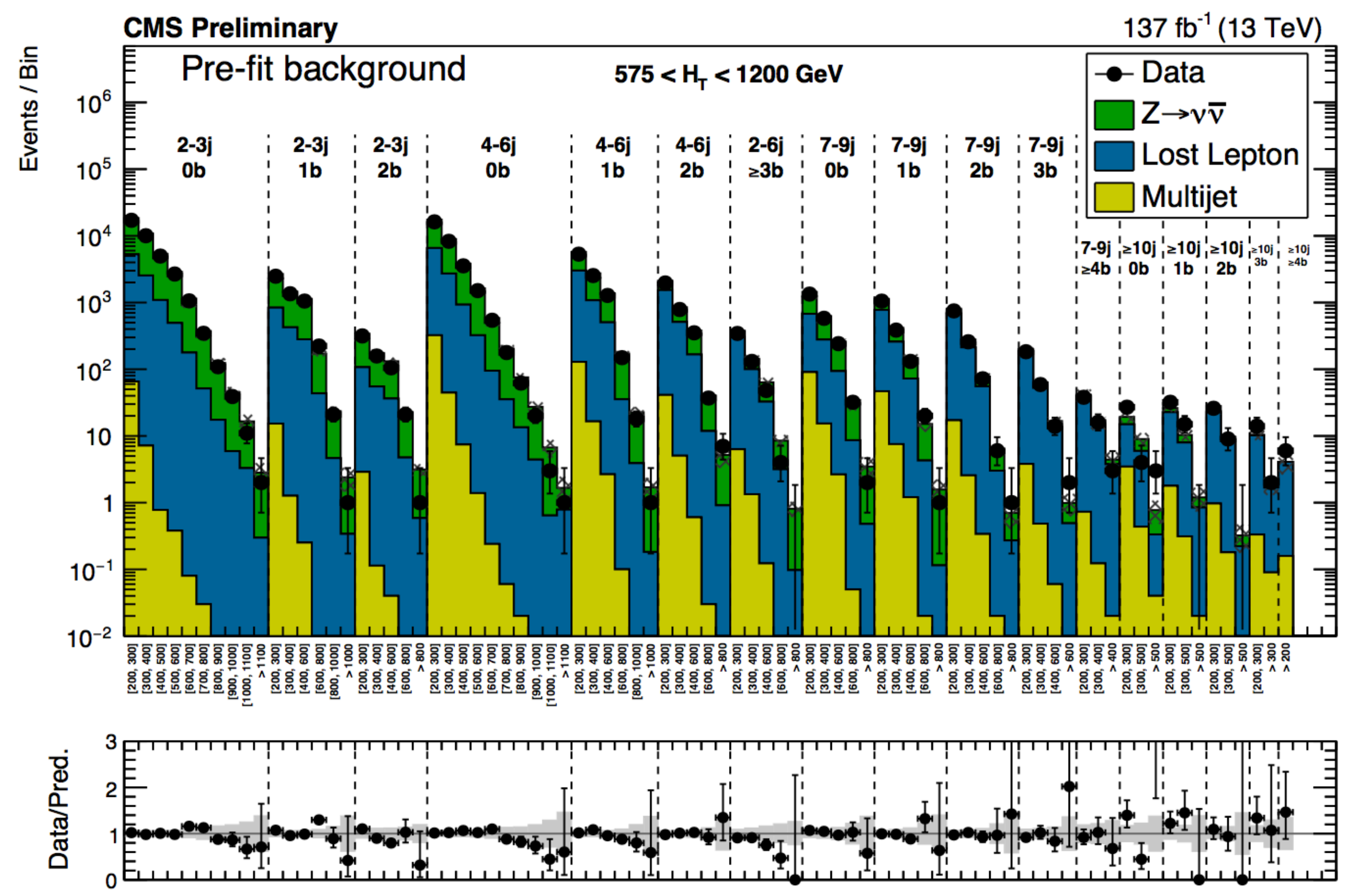


\section{Jets $+\mathrm{M}_{\mathrm{T} 2}$}
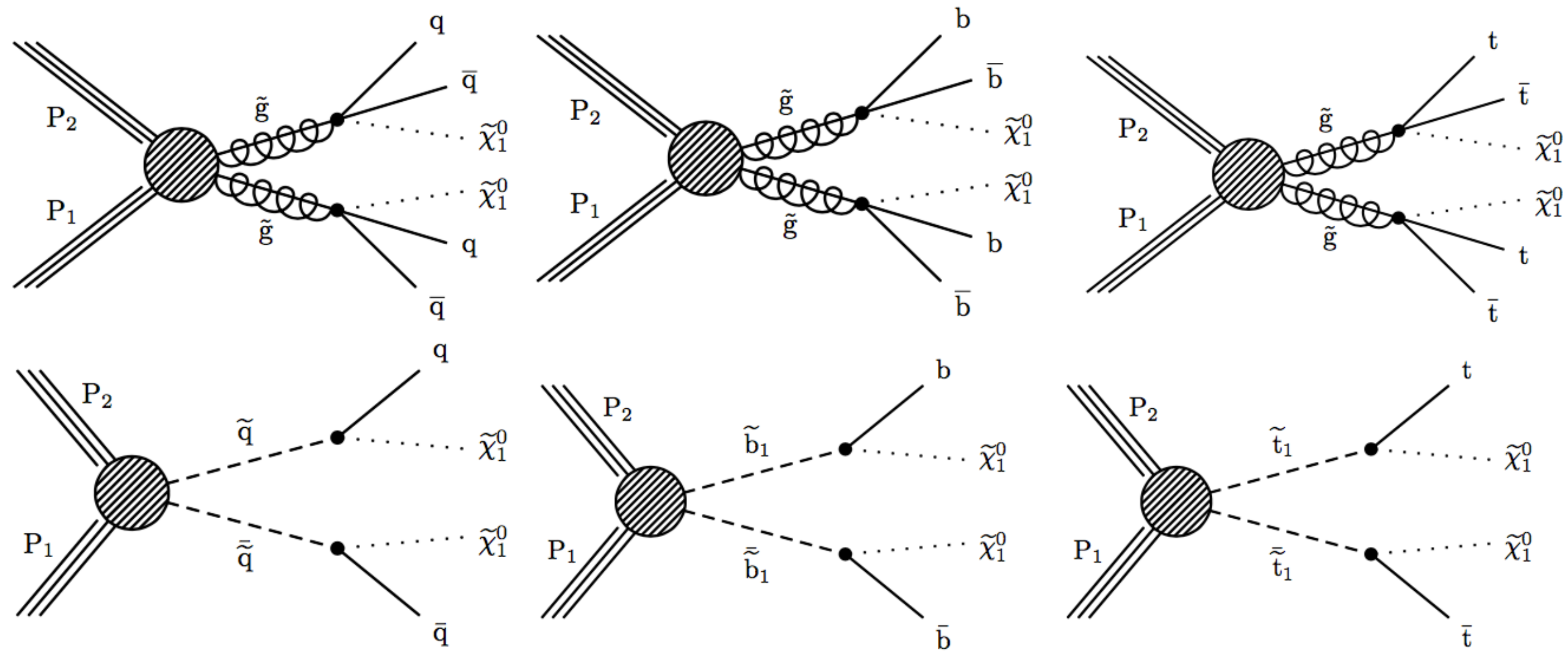


\section{Jets $+\mathrm{M}_{\mathrm{T} 2}$}

Table 2: Typical values of the systematic uncertainties as evaluated for the simplified models of SUSY used in the context of this search. The high statistical uncertainty in the simulated signal sample corresponds to a small number of signal bins with low acceptance, which are typically not among the most sensitive signal bins to that model point.

\begin{tabular}{lc}
\hline Source & Range [\%] \\
\hline Integrated luminosity & $2.3-2.5$ \\
Limited size of MC samples & $1-100$ \\
Renormalization and factorization scales & 5 \\
ISR modeling & $0-30$ \\
b tagging efficiency, heavy flavors & $0-40$ \\
b tagging efficiency, light flavors & $0-20$ \\
Lepton efficiency & $0-20$ \\
Jet energy scale & 5 \\
Fast simulation $p_{\mathrm{T}}^{\text {miss }}$ modeling & $0-5$ \\
\hline
\end{tabular}




\section{Jets $+\mathrm{M}_{\mathrm{T} 2}$}
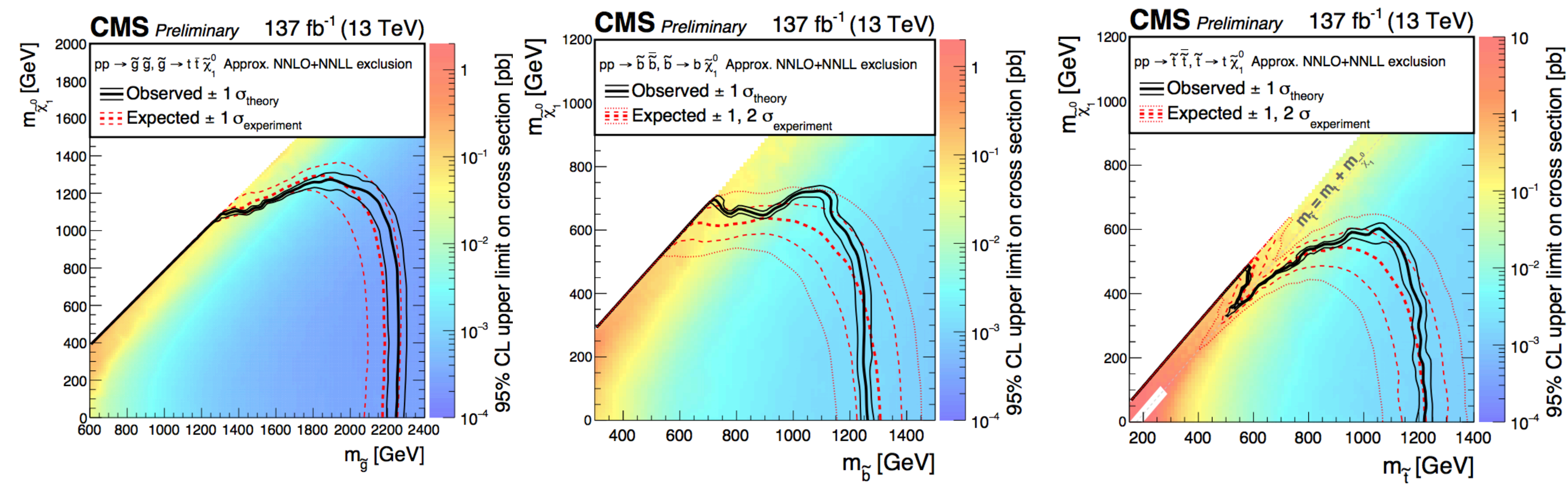


\section{bjets $+\mathrm{H}+\mathrm{p}_{T^{\text {miss }}}$}
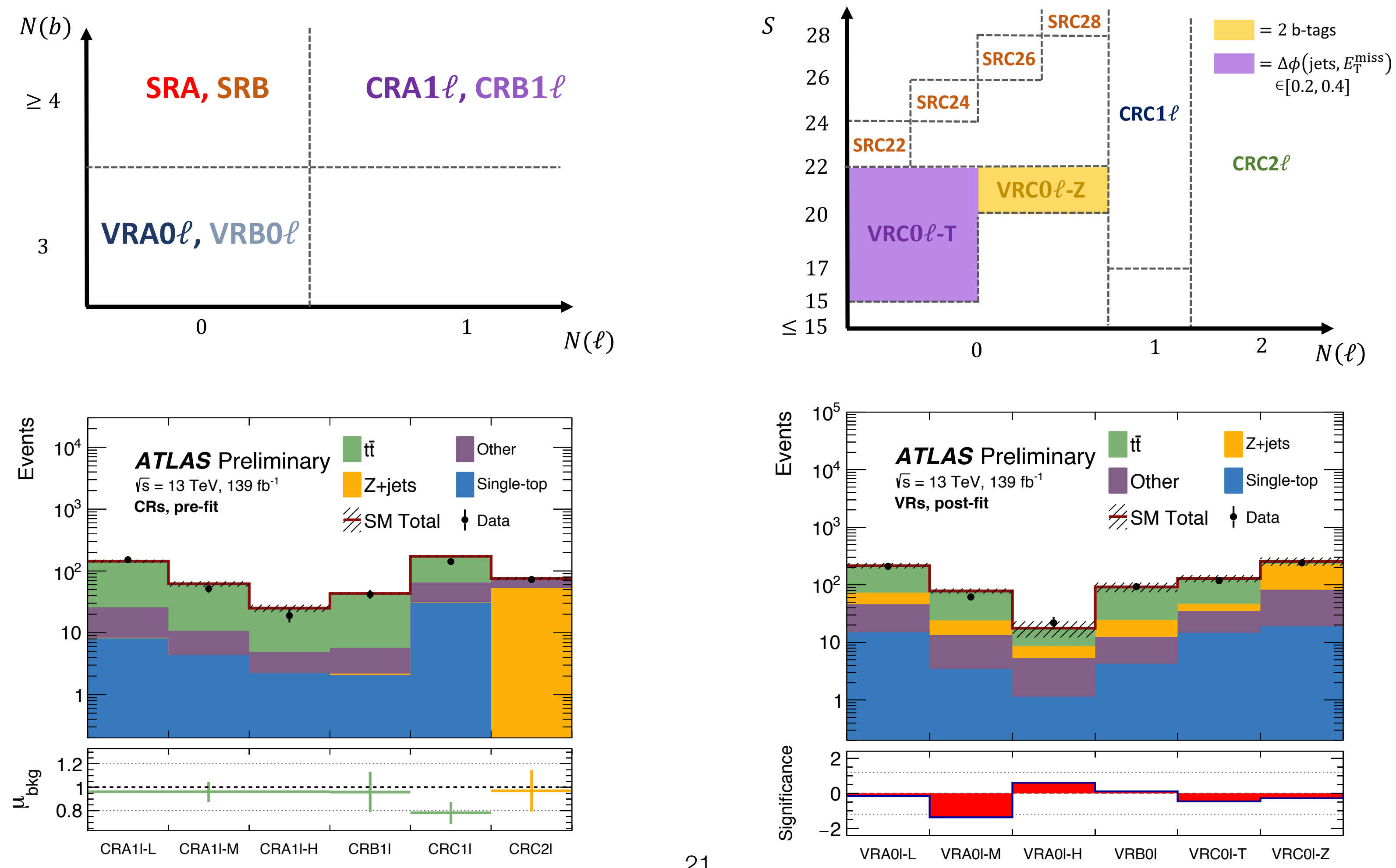


\section{bjets $+\mathrm{H}+\mathrm{p}_{T^{\text {miss }}}$}

\begin{tabular}{|c|c|c|c|c|}
\hline Variable & SRA & SRA-L & SRA-M & SRA-H \\
\hline$N_{\text {leptons }}$ (baseline) & $=0$ & & $=0$ & \\
\hline$N_{\text {jets }}$ & $\geq 6$ & & $\geq 6$ & \\
\hline$N_{\mathrm{b}-\text { jets }}$ & $\geq 4$ & & $\geq 4$ & \\
\hline$E_{\mathrm{T}}^{\mathrm{miss}}[\mathrm{GeV}]$ & $>350$ & & $>350$ & \\
\hline $\min \Delta \phi\left(\mathrm{jet}_{1-4}, \mathbf{p}_{\mathrm{T}}^{\mathrm{miss}}\right)[\mathrm{rad}]$ & $>0.4$ & & $>0.4$ & \\
\hline$\tau$ veto & Yes & & Yes & \\
\hline$p_{\mathrm{T}}\left(b_{1}\right)[\mathrm{GeV}]$ & $>200$ & & $>200$ & \\
\hline$\Delta R_{\max }(b, b)$ & ¿ 2.5 & & ¿. 2.5 & \\
\hline$\Delta R_{\max -\min }(b, b)$ & i 2.5 & & i 2.5 & \\
\hline$m\left(h_{\text {cand }}\right)[\mathrm{GeV}]$ & $>80$ & & $>80$ & \\
\hline$m_{\mathrm{eff}}[\mathrm{TeV}]$ & $>1.0$ & $\in[1.0,1.5]$ & $\in[1.5,2.0]$ & $>2.0$ \\
\hline
\end{tabular}

\begin{tabular}{lc}
\hline Variable & SRB \\
\hline$N_{\text {leptons }}($ baseline $)$ & $=0$ \\
$N_{\text {jets }}$ & $\geq 5$ \\
$N_{\mathrm{b}-\text { jets }}$ & $>4$ \\
$E_{\mathrm{T}}^{\text {miss }}[\mathrm{GeV}]$ & $>0.4$ \\
min $\Delta \phi\left(\right.$ jet $\left._{1-4}, \mathbf{p}_{\mathrm{T}}^{\text {miss }}\right)[\mathrm{rad}]$ & Yes \\
$\tau$ veto & Yes \\
$m\left(h_{\text {cand1 }}, h_{\text {cand } 2}\right)_{\text {avg }}[\mathrm{GeV}]$ & $\in[5,175]$ \\
leading jet not $b$-tagged & $>350$ \\
$p_{\mathrm{T}}\left(j_{1}\right)[\mathrm{GeV}]$ & $>2.8$ \\
$\left|\Delta \phi\left(j_{1}, E_{\mathrm{T}}^{\text {miss }}\right)\right|[\mathrm{rad}]$ & $>1$ \\
$m_{\text {eff }}[\mathrm{TeV}]$ &
\end{tabular}

\begin{tabular}{|c|c|c|c|c|c|}
\hline Variable & $\mathrm{SRC}$ & SRC22 & $\mathrm{SRC} 24$ & SRC26 & $\mathrm{SRC} 28$ \\
\hline$N_{\text {leptons }}$ (baseline) & $=0$ & \multicolumn{4}{|c|}{$=0$} \\
\hline$N_{\text {jets }}$ & $\geq 4$ & \multicolumn{4}{|c|}{$\geq 4$} \\
\hline$N_{\text {b-jets }}$ & $\geq 3$ & \multicolumn{4}{|c|}{$\geq 3$} \\
\hline$E_{\mathrm{T}}^{\text {miss }}[\mathrm{GeV}]$ & $>250$ & \multicolumn{4}{|c|}{$>250$} \\
\hline $\min \Delta \phi\left(\right.$ jet $\left._{1-4}, \mathbf{p}_{\mathrm{T}}^{\mathrm{miss}}\right)[\mathrm{rad}]$ & $>0.4$ & \multicolumn{4}{|c|}{$>0.4$} \\
\hline $\mathcal{S}$ & $>22$ & $\in[22,24]$ & $\in[24,26]$ & $\in[26,28]$ & $>28$ \\
\hline
\end{tabular}




\section{SS + multilepton}

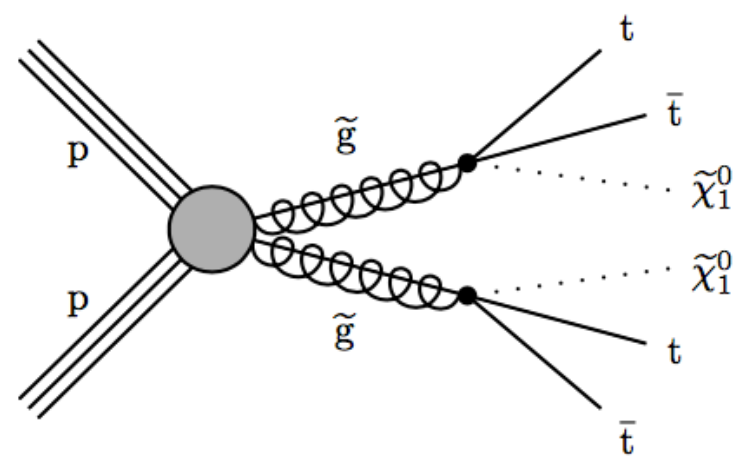

(a) T1tttt

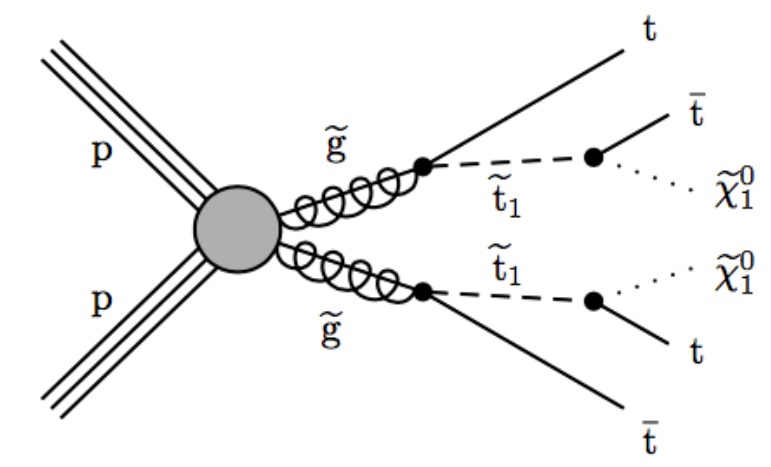

(b) T5tttt

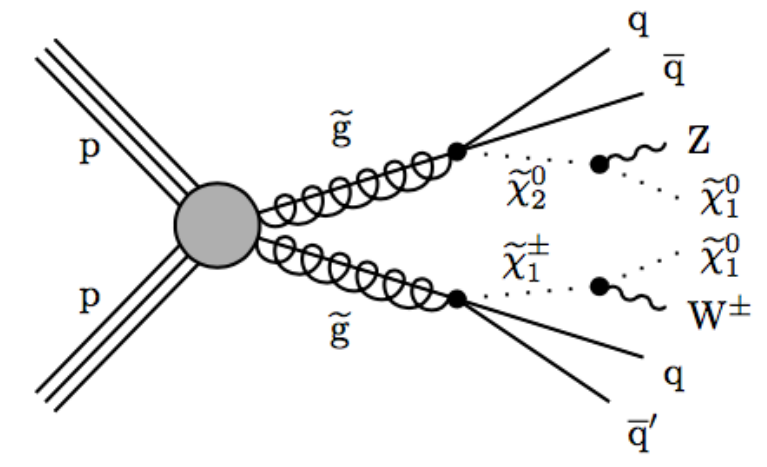

(c) T5qqqqWZ

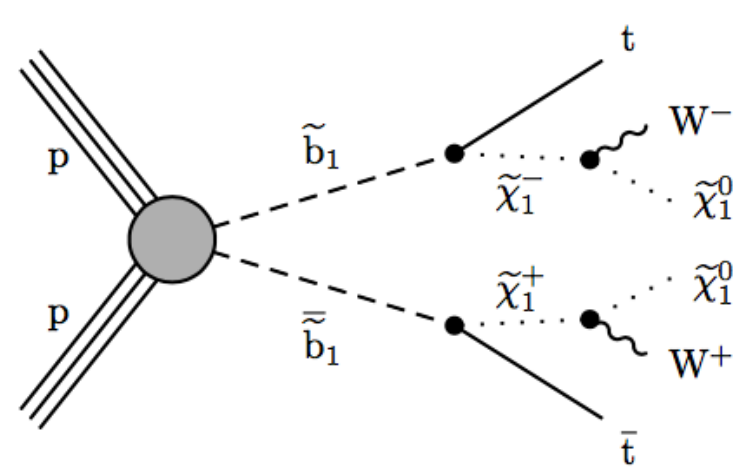

(d) T6ttWW

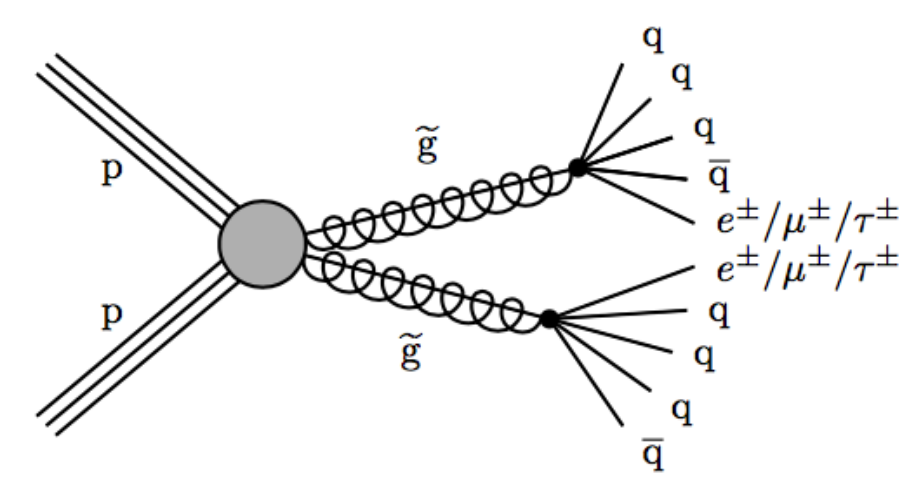

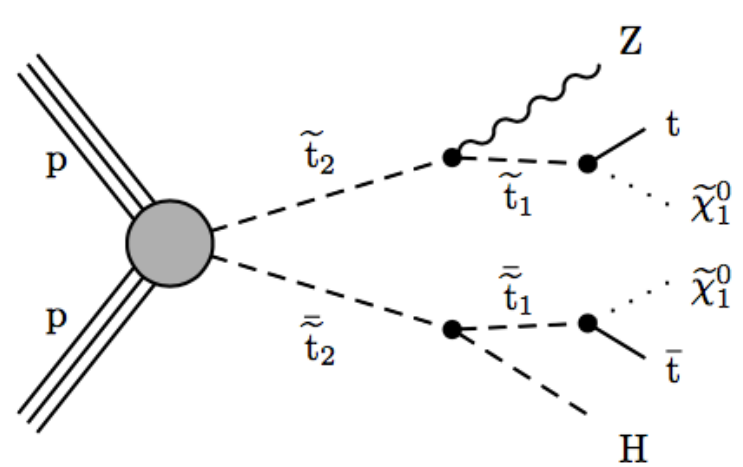

(e) T6ttHZ

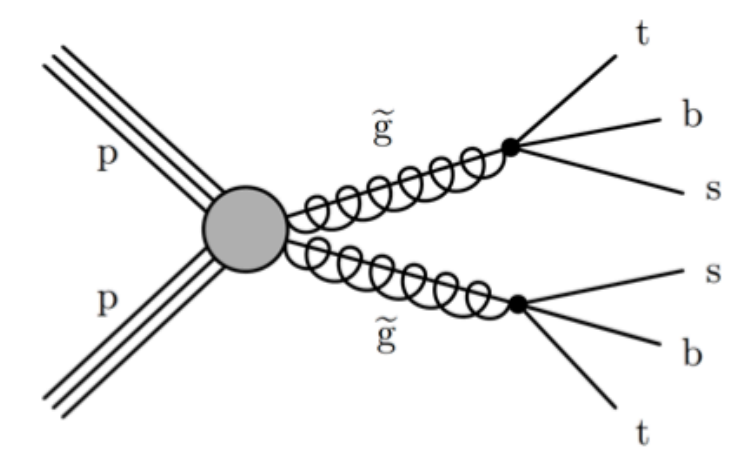




\section{SS + multilepton}

Six exclusive categories are then built:

- SSHH: exactly 2 leptons, both with $p_{\mathrm{T}}>25 \mathrm{GeV}$, and $p_{\mathrm{T}}^{\text {miss }}>50 \mathrm{GeV}$.

- SSHL: exactly 2 leptons, one with $p_{\mathrm{T}}>25 \mathrm{GeV}$, one with $p_{\mathrm{T}}<25 \mathrm{GeV}$ and $p_{\mathrm{T}}^{\text {miss }}>$ $50 \mathrm{GeV}$.

- SSLL: exactly 2 leptons, both with $p_{\mathrm{T}}<25 \mathrm{GeV}$ and $p_{\mathrm{T}}^{\text {miss }}>50 \mathrm{GeV}$.

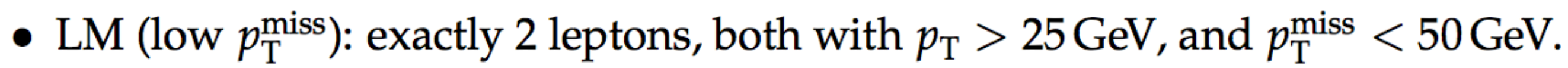

- ML (multilepton): $\geq 3$ leptons, at least one with $p_{\mathrm{T}}>25 \mathrm{GeV}, p_{\mathrm{T}}^{\text {miss }}>50 \mathrm{GeV}$.
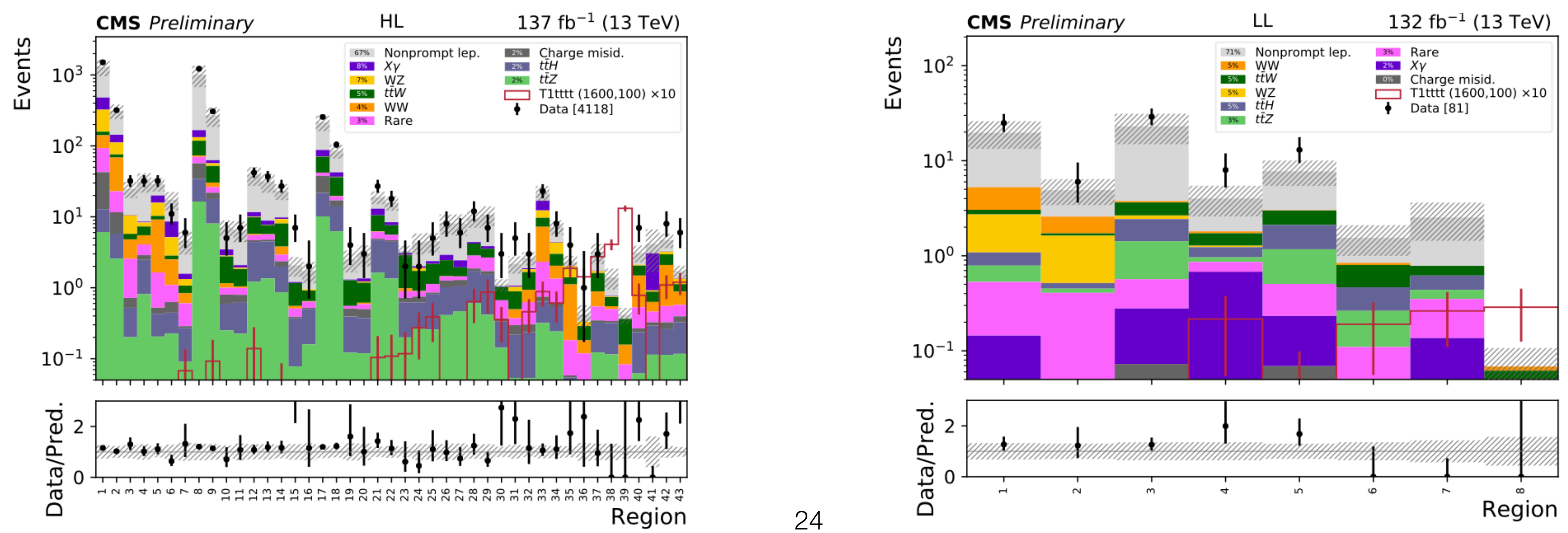


\section{SS + multilepton}

Six exclusive categories are then built:

- SSHH: exactly 2 leptons, both with $p_{\mathrm{T}}>25 \mathrm{GeV}$, and $p_{\mathrm{T}}^{\text {miss }}>50 \mathrm{GeV}$.

- SSHL: exactly 2 leptons, one with $p_{\mathrm{T}}>25 \mathrm{GeV}$, one with $p_{\mathrm{T}}<25 \mathrm{GeV}$ and $p_{\mathrm{T}}^{\text {miss }}>$ $50 \mathrm{GeV}$.

- SSLL: exactly 2 leptons, both with $p_{\mathrm{T}}<25 \mathrm{GeV}$ and $p_{\mathrm{T}}^{\text {miss }}>50 \mathrm{GeV}$.

- LM (low $p_{\mathrm{T}}^{\text {miss }}$ ): exactly 2 leptons, both with $p_{\mathrm{T}}>25 \mathrm{GeV}$, and $p_{\mathrm{T}}^{\text {miss }}<50 \mathrm{GeV}$.

- ML (multilepton): $\geq 3$ leptons, at least one with $p_{\mathrm{T}}>25 \mathrm{GeV}, p_{\mathrm{T}}^{\text {miss }}>50 \mathrm{GeV}$.
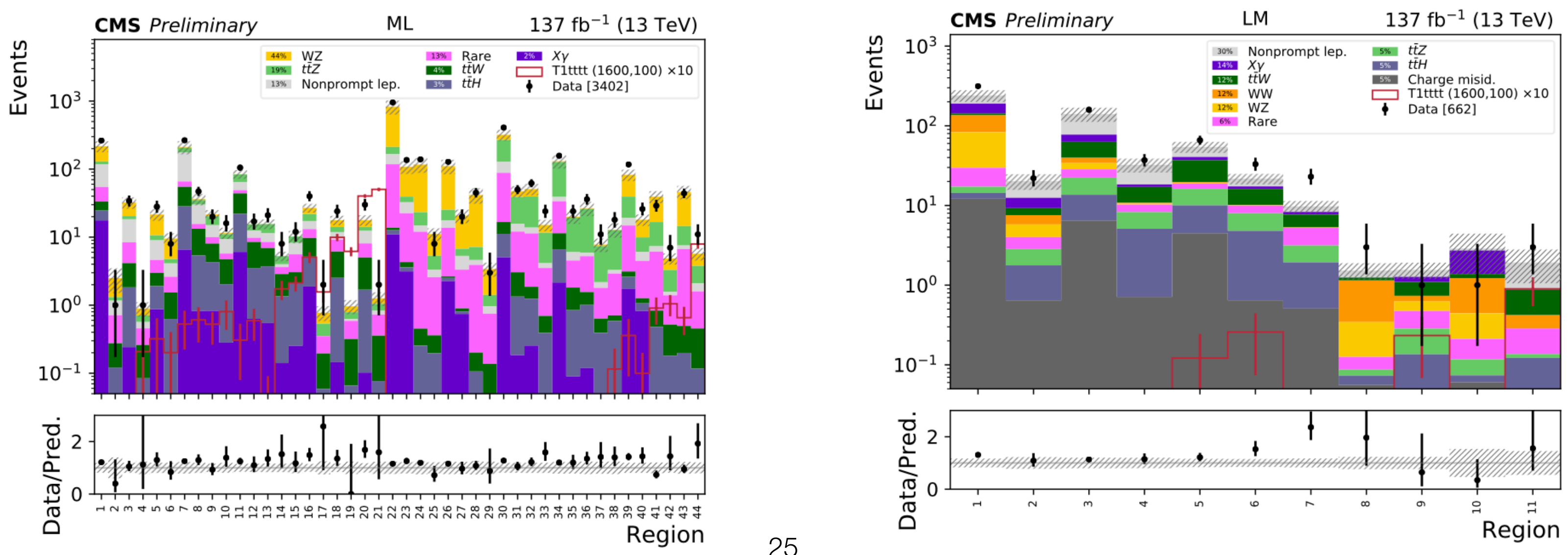


\section{SS + multilepton}
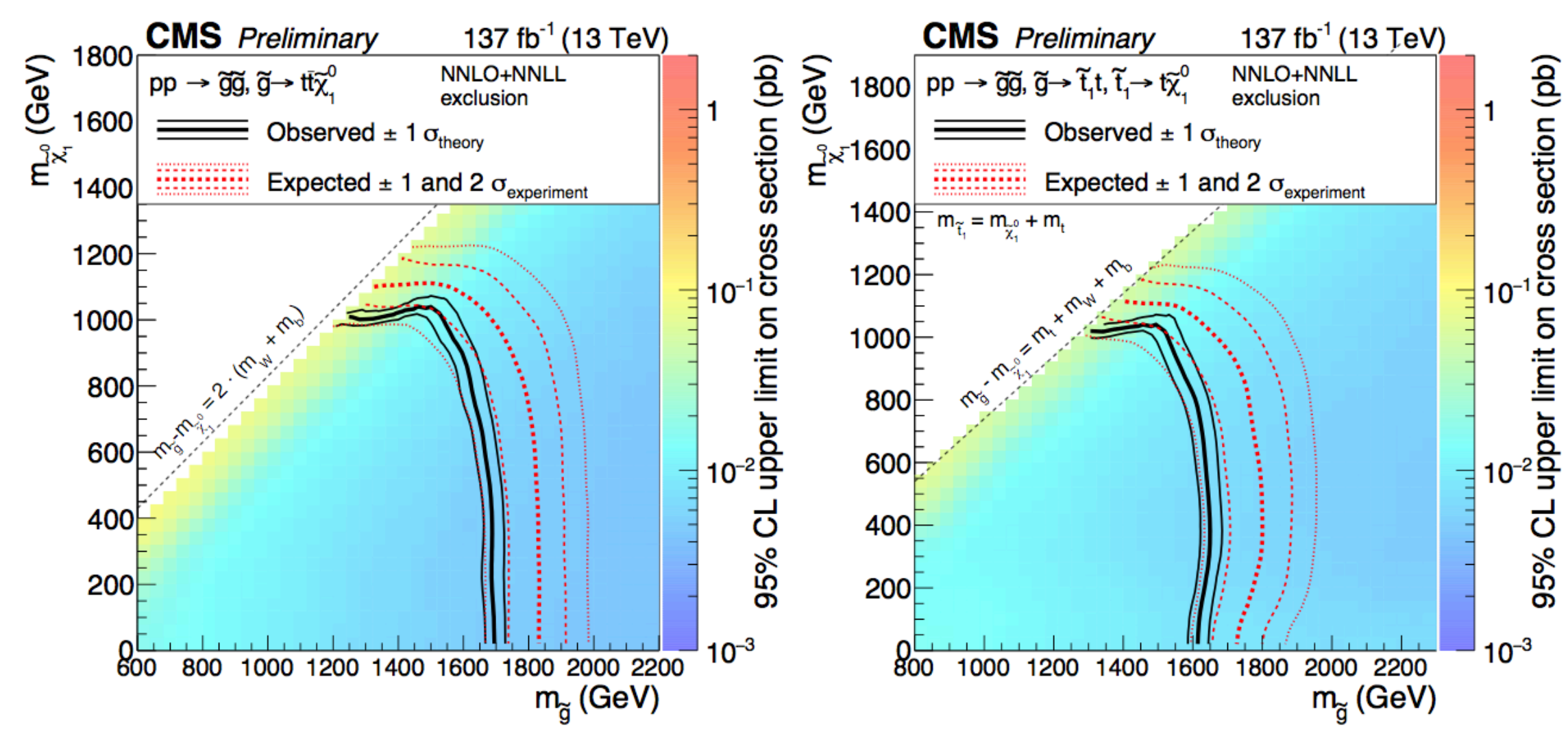

CMS Preliminary

$137 \mathrm{fb}^{-1}$ (13 TeV)
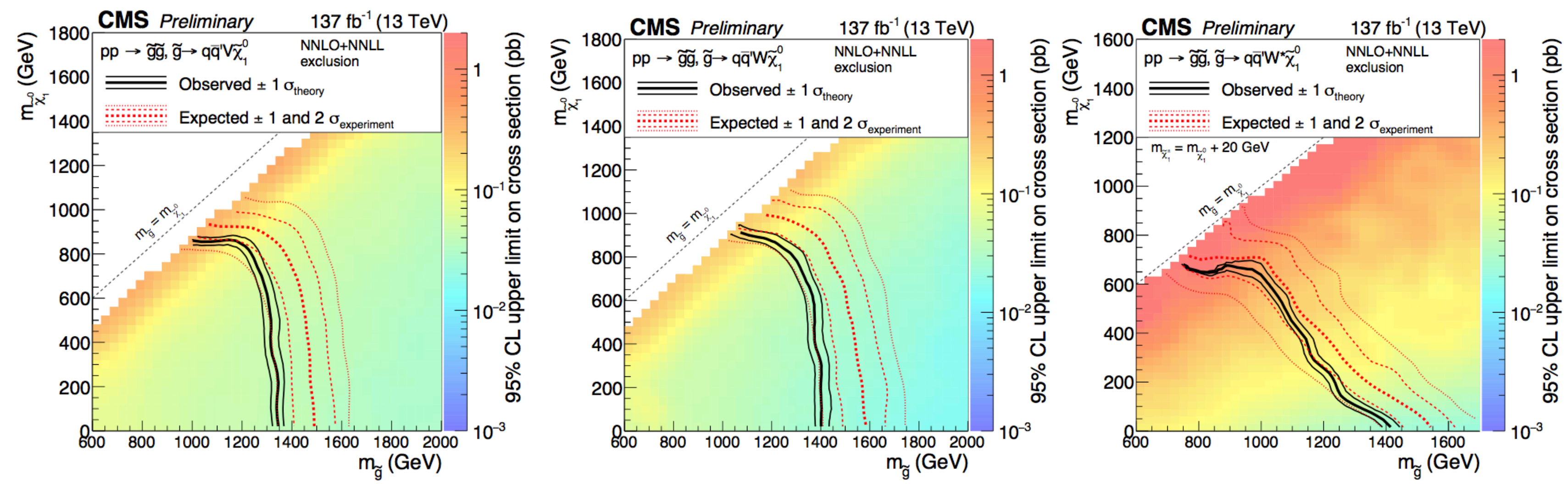


\section{SS + multilepton}

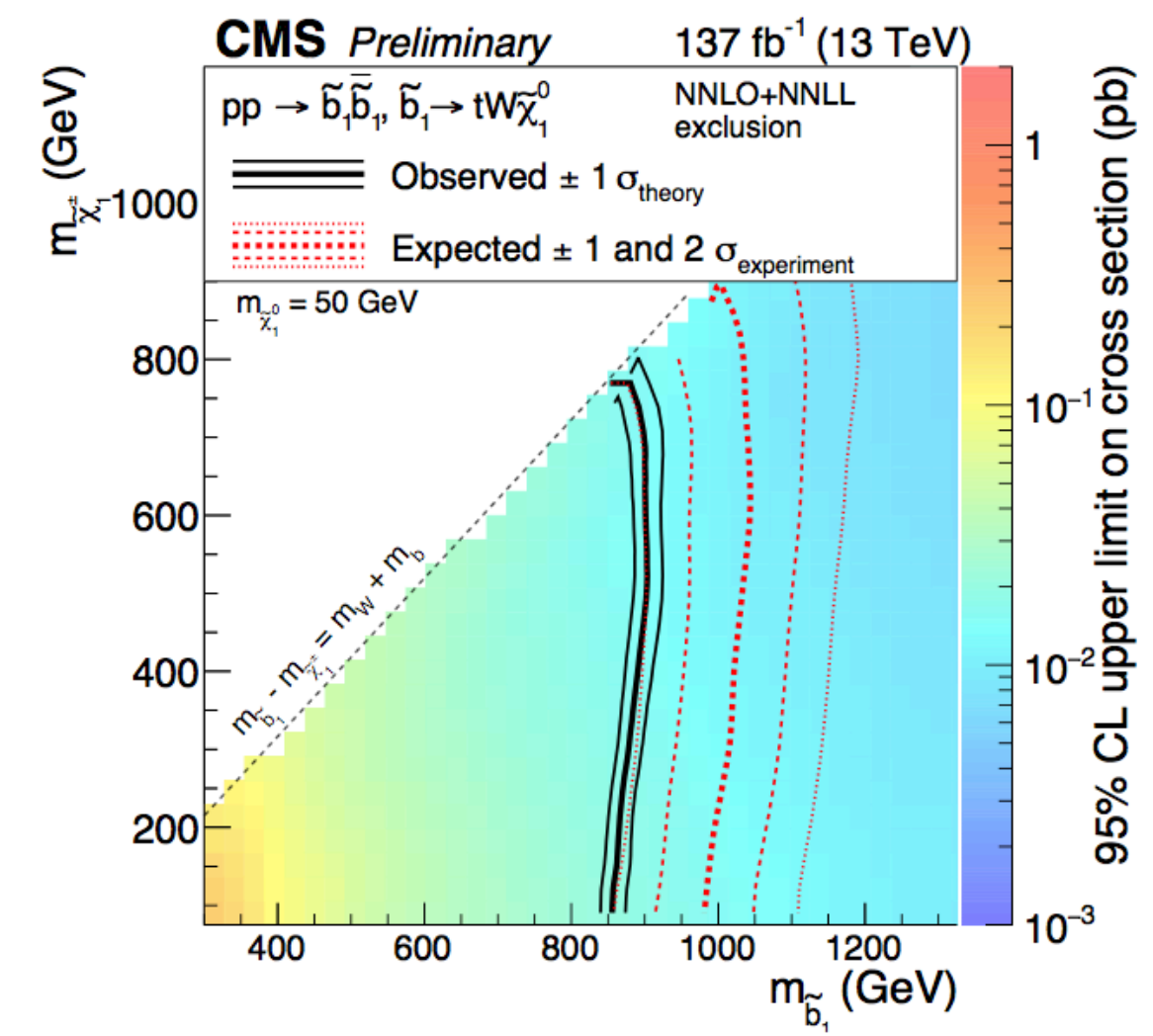

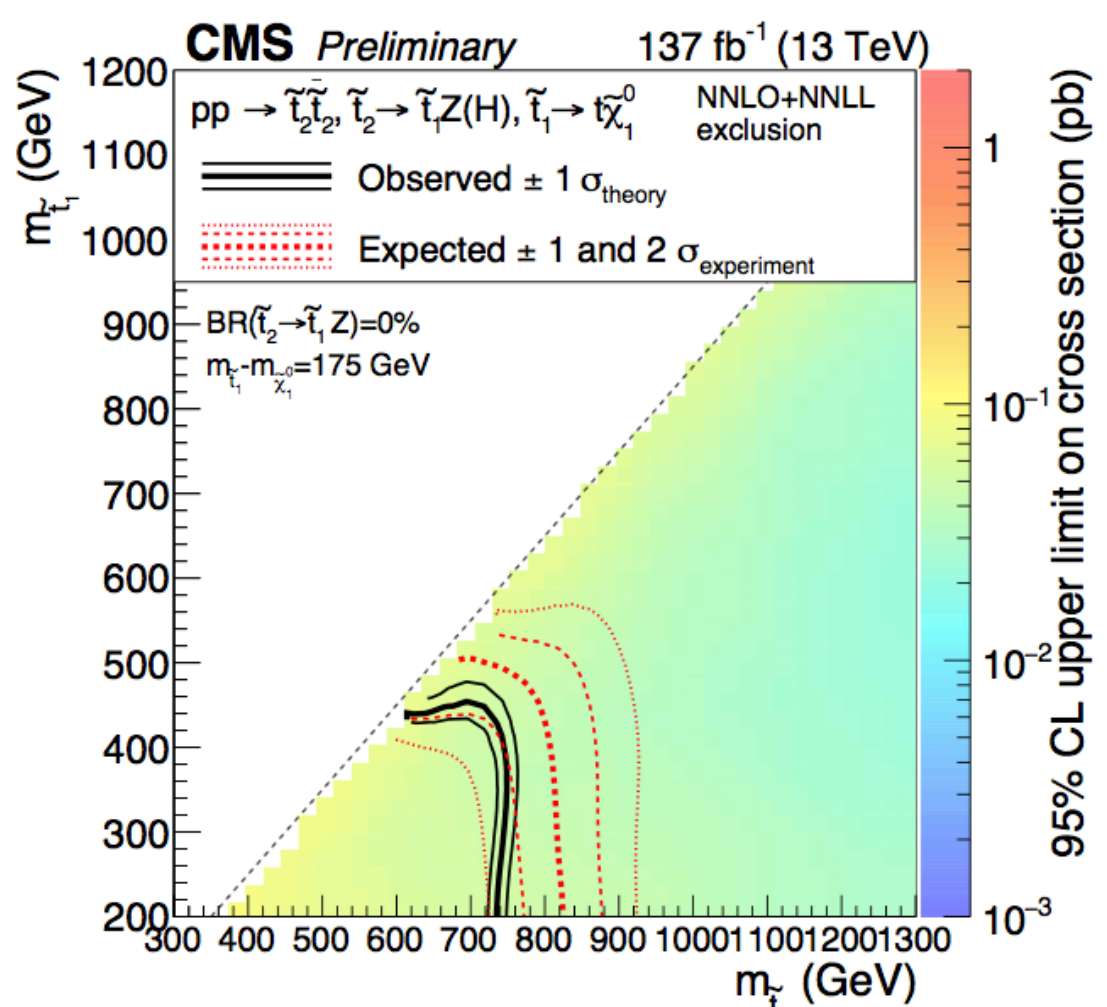

$m_{t_{5}}(\mathrm{GeV})$

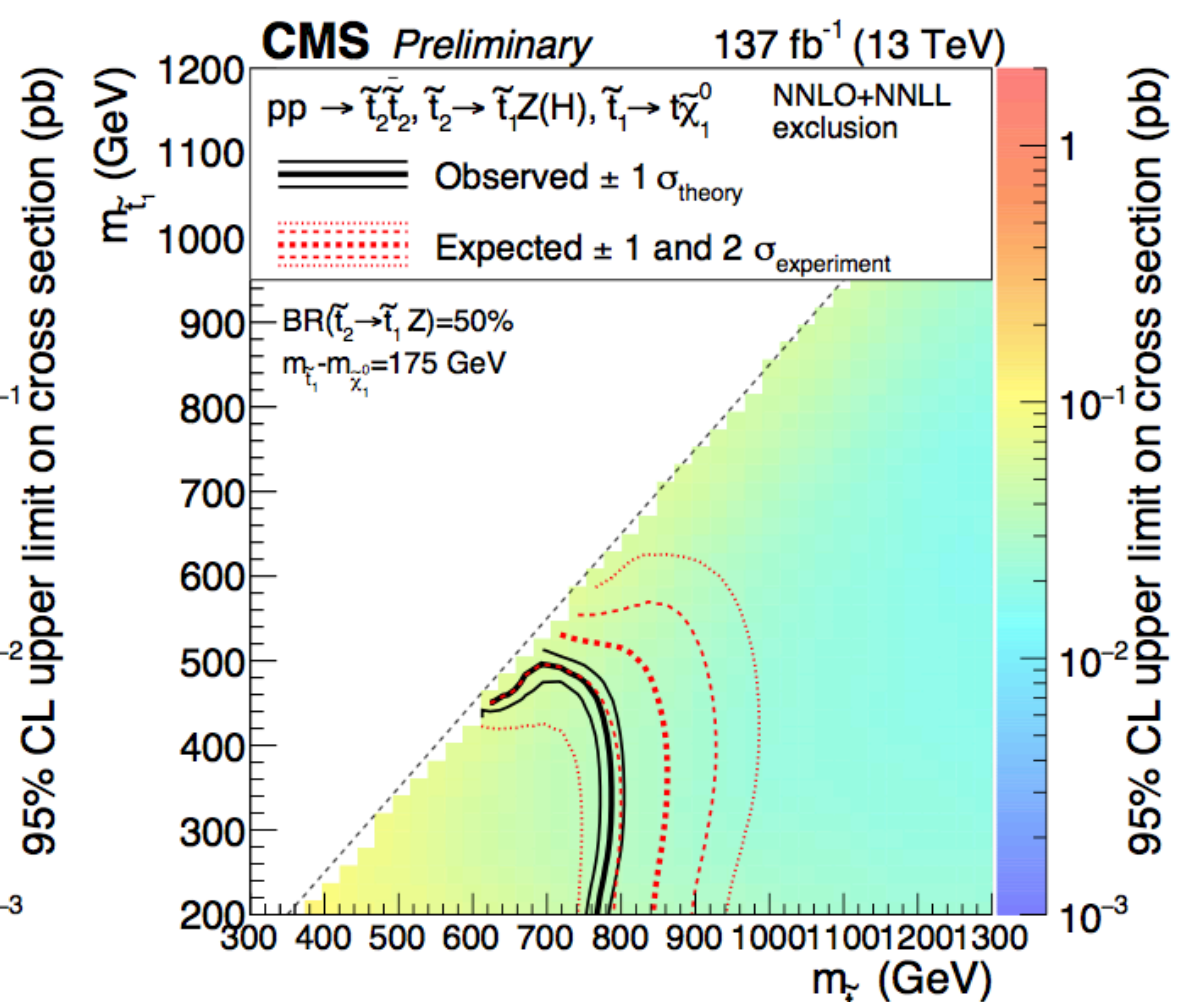

$m_{\mathrm{t}_{2}}(\mathrm{GeV})$ 


\section{SS + multilepton}

Table 7: Summary of the sources of uncertainty and their effect on the yields of different processes in the SRs. The first two groups list experimental and theoretical uncertainties assigned to processes estimated using simulation, while the last group lists uncertainties assigned to processes whose yield is estimated from data. The uncertainties in the first group also apply to signal samples. Reported values are representative for the most relevant signal regions.

\begin{tabular}{l|c|c}
\hline Source & Typical uncertainty (\%) & Correlation across years \\
\hline \hline Integrated luminosity & $2.3-2.5$ & Uncorrelated \\
Lepton selection & $2-10$ & Uncorrelated \\
Trigger efficiency & $2-7$ & Uncorrelated \\
Pileup & $0-6$ & Uncorrelated \\
Jet energy scale & $1-15$ & Uncorrelated \\
b tagging & $1-10$ & Uncorrelated \\
Simulated sample size & $1-20$ & Uncorrelated \\
\hline Scale and PDF variations & $10-20$ & Correlated \\
Theoretical background cross sections & $30-50$ & Correlated \\
\hline Nonprompt leptons & 30 & Correlated \\
Charge misidentification & 20 & Uncorrelated \\
\hline
\end{tabular}




\section{Stop with charm tagging}

- A "tight" working point was adopted in the analysis, resulting in a charm-tagging efficiency of about $18 \%$, a b-jet rejection factor of 20 , a light-flavour jet rejection factor of 200 , and a hadronic $\tau$ jet rejection factor of 6 ,

$$
m_{\mathrm{T}}^{c}=\min _{c \text {-jets }} \sqrt{2 \cdot E_{\mathrm{T}}^{\text {miss }} p_{\mathrm{T}}^{c} \cdot\left(1-\cos \Delta \phi\left(\boldsymbol{E}_{\mathrm{T}}^{\text {miss }}, \boldsymbol{p}_{\mathrm{T}}^{c}\right)\right)},
$$

\begin{tabular}{|c|c|c|c|c|c|}
\hline & SR1 & SR2 & SR3 & SR4 & SR5 \\
\hline Trigger & \multicolumn{5}{|c|}{$E_{\mathrm{T}}^{\mathrm{miss}}$ triggers } \\
\hline Leptons & \multicolumn{5}{|c|}{$0 e \operatorname{AND} 0 \mu$} \\
\hline$E_{\mathrm{T}}^{\mathrm{miss}}[\mathrm{GeV}]$ & \multicolumn{5}{|c|}{$>500$} \\
\hline$\Delta \phi_{\min }\left(\mathrm{jet}, \boldsymbol{E}_{\mathrm{T}}^{\mathrm{miss}}\right)[\mathrm{rad}]$ & \multicolumn{5}{|c|}{$>0.4$} \\
\hline$N_{c \text {-jets }}$ & & & 1 & & \\
\hline$N_{\text {jets }}$ & $\geq 2$ & $\geq 3$ & $\geq 3$ & $\geq 3$ & $\geq 3$ \\
\hline Leading jet $c$-tag veto & yes & yes & yes & yes & no \\
\hline$p_{\mathrm{T}}^{j_{1}}[\mathrm{GeV}]$ & $>250$ & $>250$ & $>250$ & $>250$ & $>300$ \\
\hline$p_{\mathrm{T}}^{j_{2}}[\mathrm{GeV}]$ & - & - & $>100$ & $>140$ & $>200$ \\
\hline$p_{\mathrm{T}}^{j_{3}^{3}}[\mathrm{GeV}]$ & - & - & $>80$ & $>120$ & $>150$ \\
\hline$p_{\mathrm{T}}^{c_{1}}[\mathrm{GeV}]$ & $<100$ & $>60$ & $>80$ & $>100$ & $>150$ \\
\hline$m_{\mathrm{T}}^{c}[\mathrm{GeV}]$ & $\in(120,250)$ & $\in(120,250)$ & $\in(175,400)$ & $>200$ & $>400$ \\
\hline
\end{tabular}




\section{Stop with charm tagging}

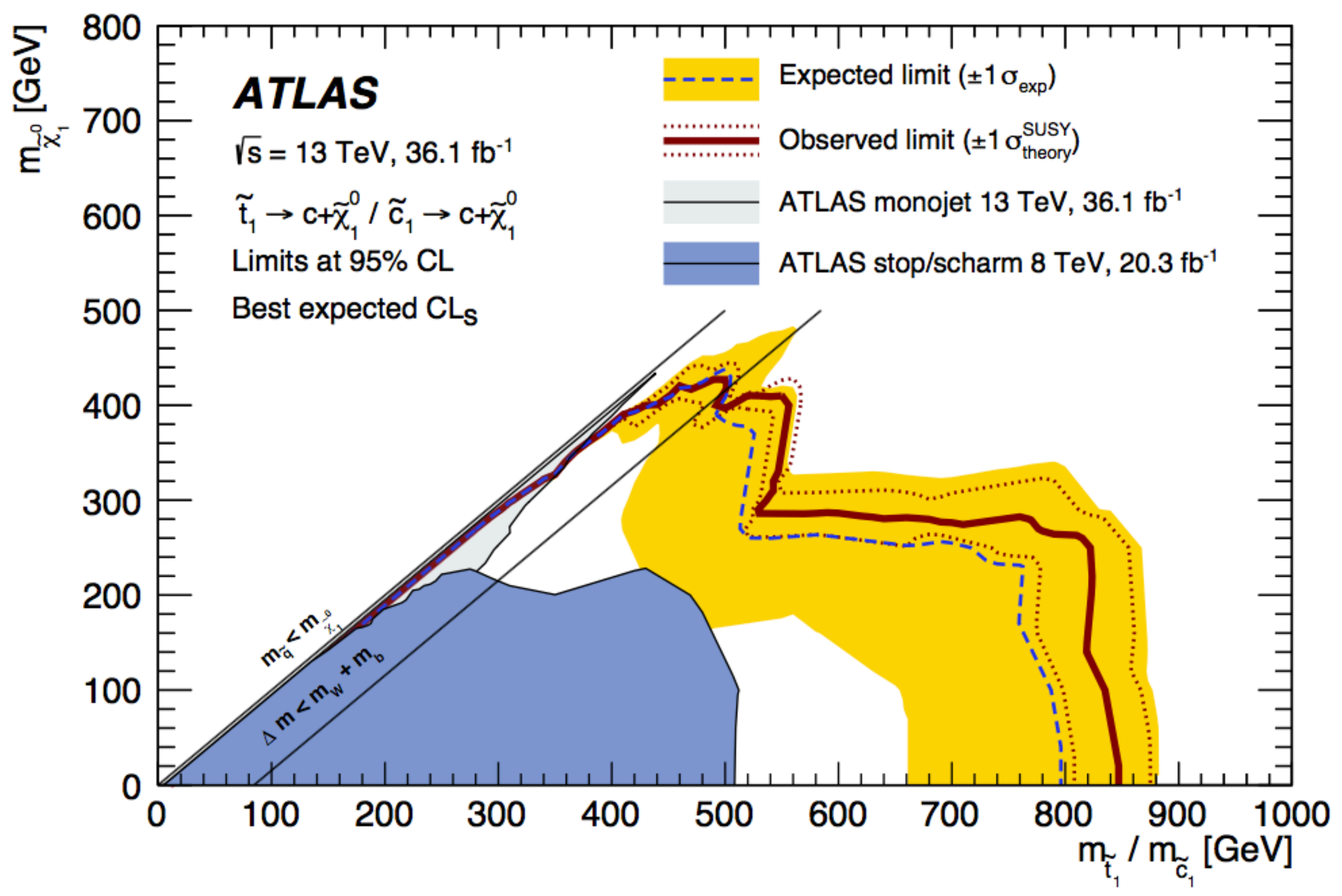




\section{Spin correlations}
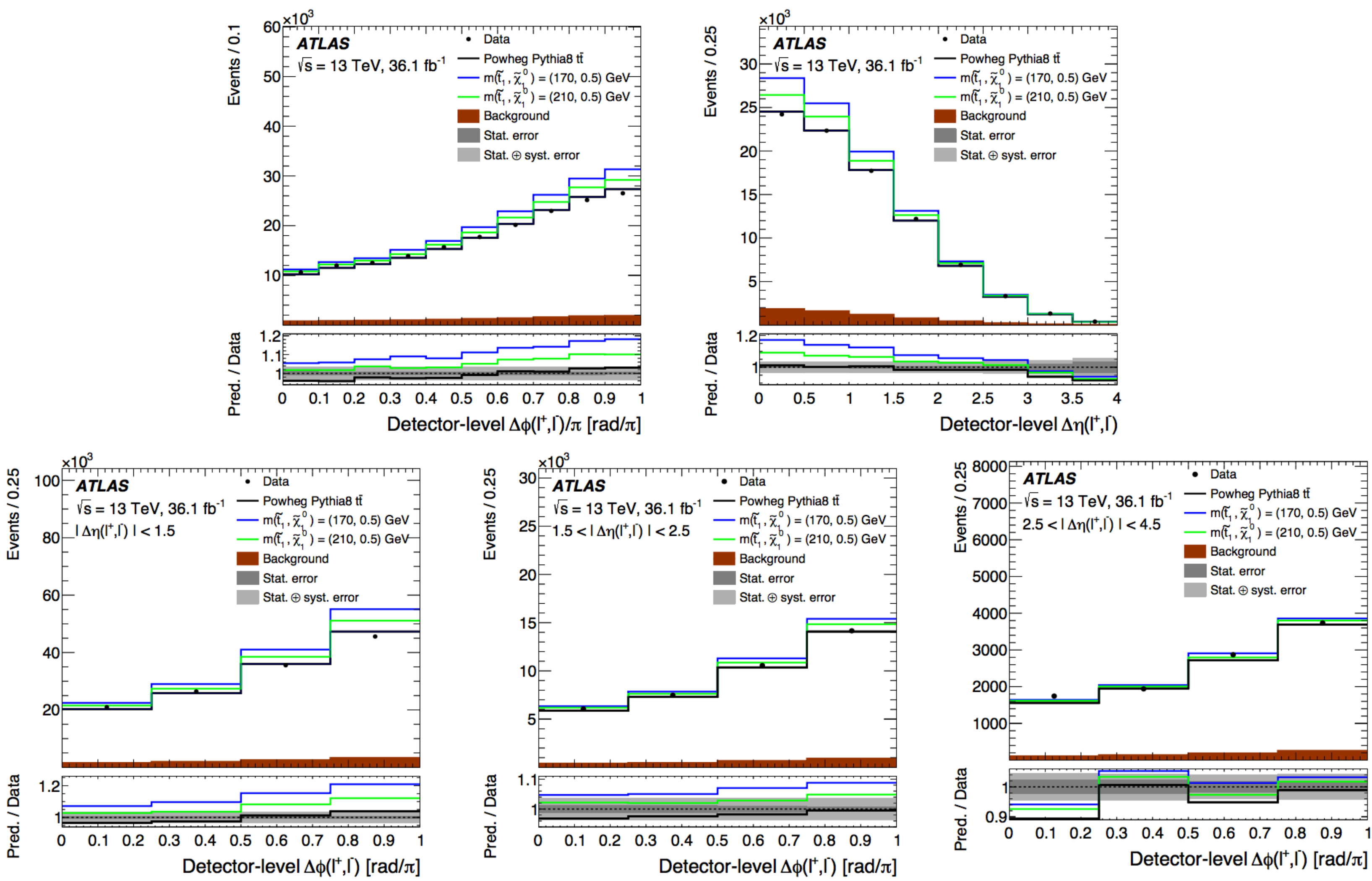


\section{Top corridor - $\mathrm{M}_{\mathrm{T} 2}$}

- The sensitivity of the analysis comes from a precise estimate of the tt background, using MC simulation and exploiting the 6\% [54] theoretical uncertainties on the predicted cross section and the even smaller [31,69] experimental uncertainties on the measurement. Additional sensitivity comes from the small kinematic differences between the target signal and the tt background, which become more important with increasing top squark mass and increasing mass difference between the top squark and neutralino.

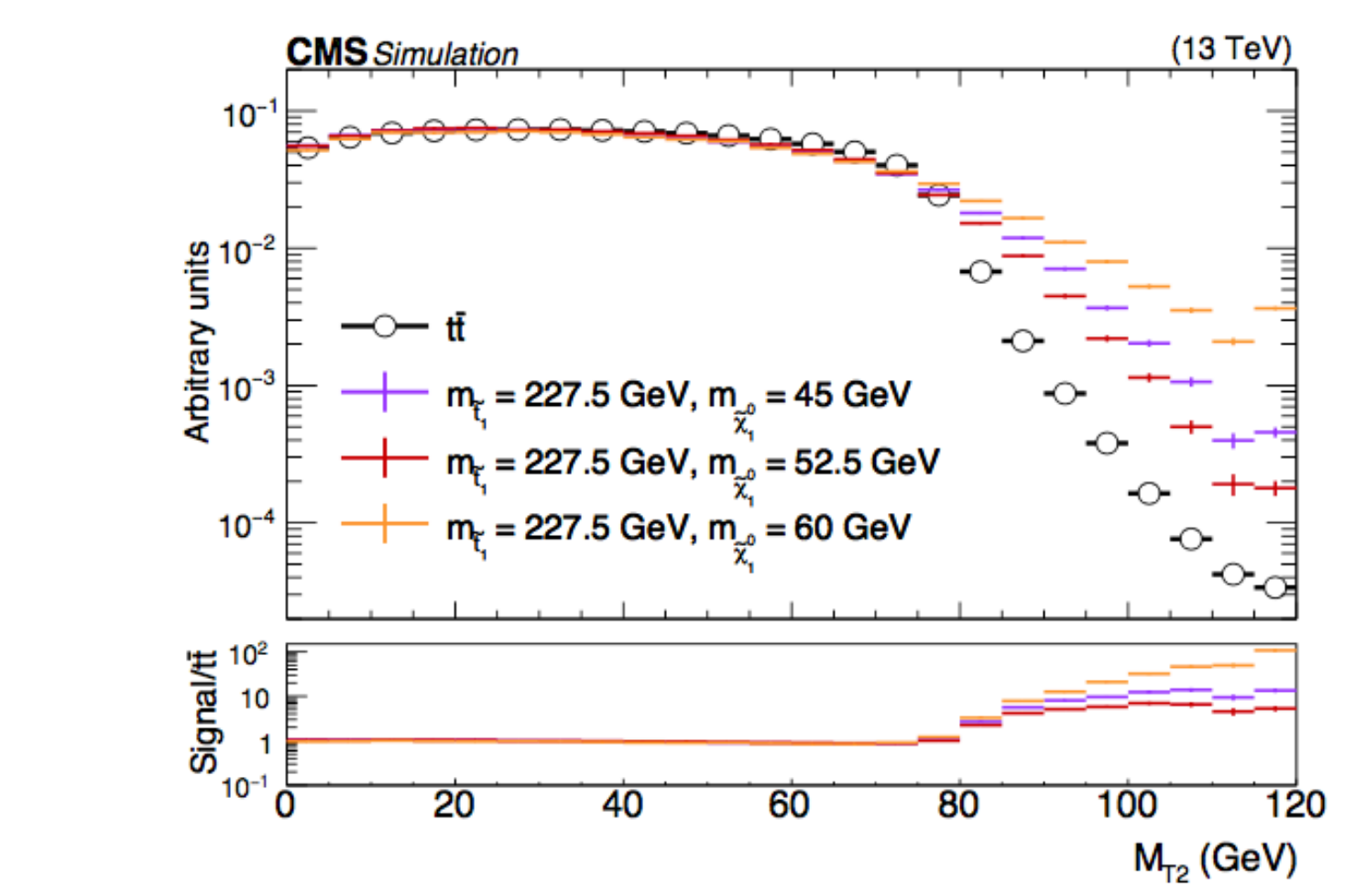

$$
M_{\mathrm{T} 2}=\min _{\vec{p}_{\mathrm{T}, 1}^{\text {miss }}+\vec{p}_{\mathrm{T}, 2}^{\text {miss }}=\vec{p}_{\mathrm{T}}^{\text {miss }}}\left(\max \left[m_{\mathrm{T}}\left(\vec{p}_{\mathrm{T}}^{\ell 1}, \vec{p}_{\mathrm{T}, 1}^{\text {miss }}\right), m_{\mathrm{T}}\left(\vec{p}_{\mathrm{T}}^{\ell 2}, \vec{p}_{\mathrm{T}, 2}^{\text {miss }}\right)\right]\right)
$$

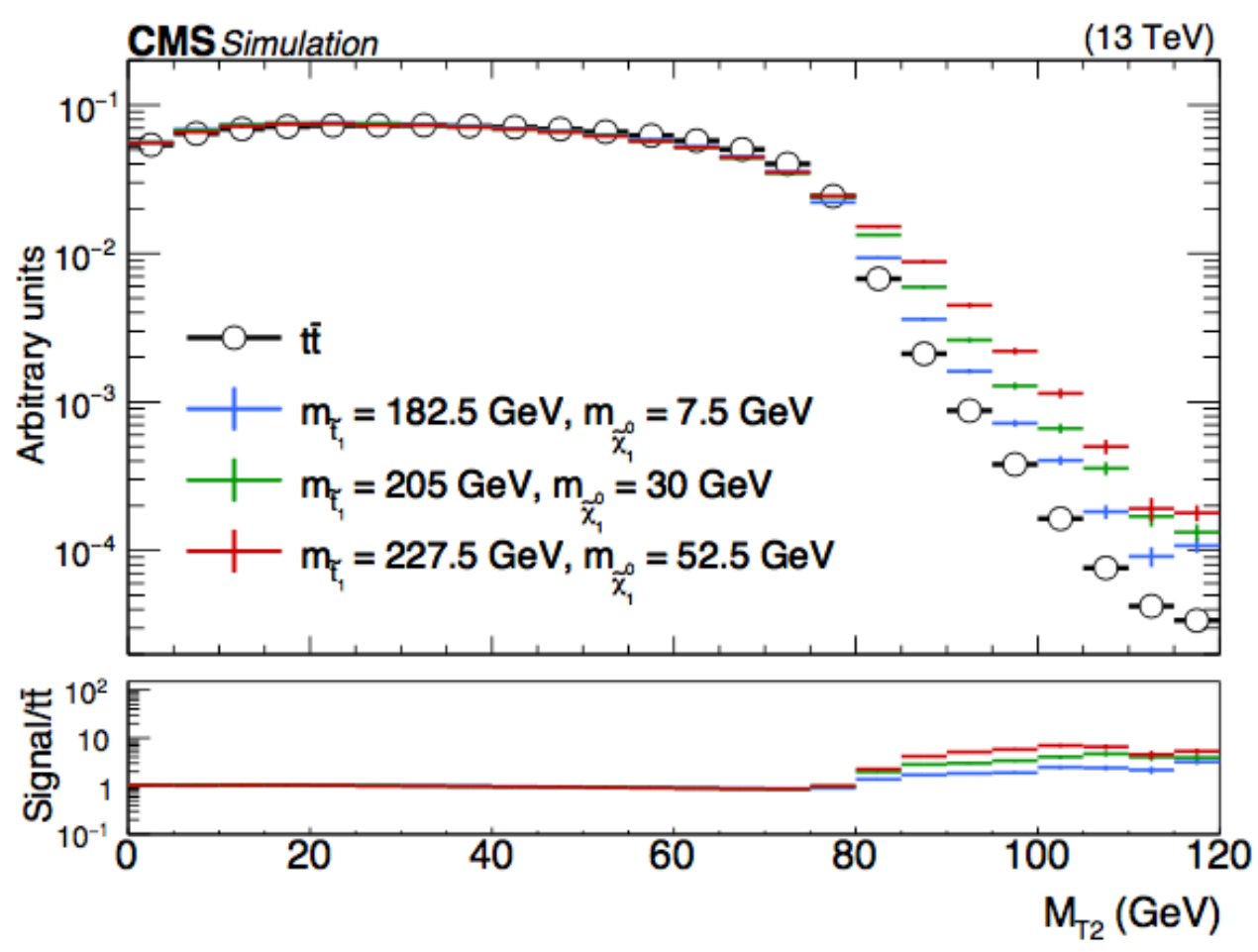




\section{Top corridor $-\mathrm{M}_{\mathrm{T} 2}$}

\begin{tabular}{lc} 
Source & Range $(\%)$ \\
\hline$\mu_{\mathrm{F}}$ and $\mu_{\mathrm{R}}$ scales & $0.3-1.0$ \\
$\mathrm{PDF}$ & $\approx 0.6$ \\
Initial-state radiation & $0.5-1.0$ \\
Final-state radiation & $0.6-1.2$ \\
ME/PS matching $\left(h_{\text {damp }}\right)$ & $0.3-2.0$ \\
Underlying event & $\approx 0.8$ \\
Colour reconnection & $\approx 1.5$ \\
Top quark $p_{\mathrm{T}}$ reweighting & $0.1-0.5$ \\
Top quark mass (acceptance) & $\approx 1.0$ \\
& \\
& \\
Source & \\
\hline Muon efficiencies & Range for $\mathrm{t \overline {t }}$ and signal (\%) \\
Electron efficiencies & $\approx 1.4$ \\
Trigger efficiency & $\approx 1.5$ \\
Lepton energy scale & $\approx 0.6$ \\
Jet energy scale & $0.5-2.0$ \\
Jet energy resolution & $1.5-3.0$ \\
btagging efficiency & $0.3-3.5$ \\
Mistag efficiency & $1.2-2.0$ \\
Unclustered energy & $0.2-0.6$ \\
Pileup & $0.5-1.5$
\end{tabular}

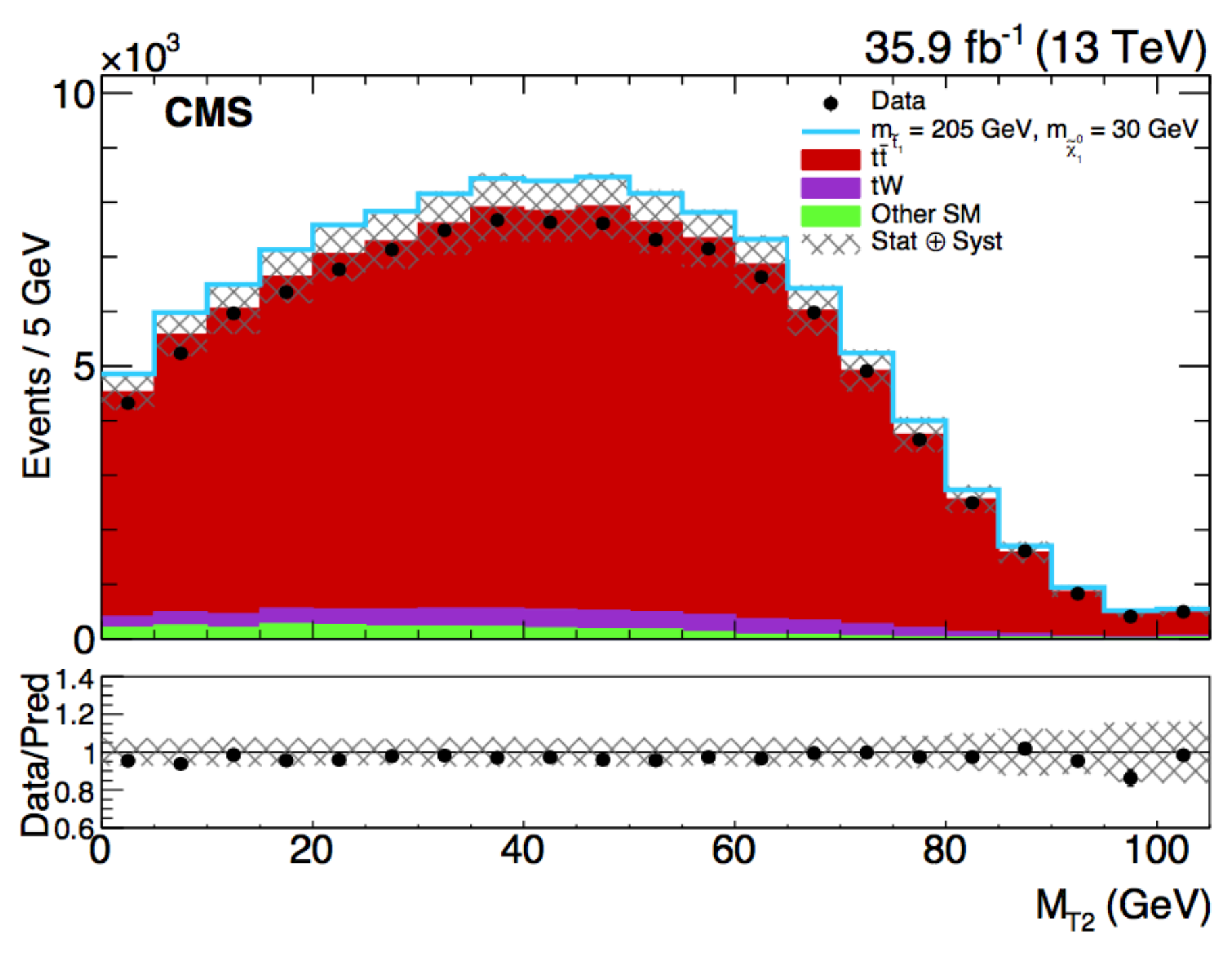

